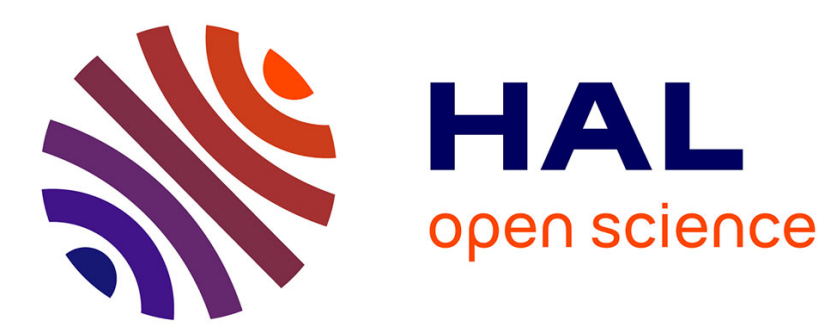

\title{
A computational linear elastic fracture mechanics-based model for alkali-silica reaction
}

Laurent Charpin, Alain Ehrlacher

\section{To cite this version:}

Laurent Charpin, Alain Ehrlacher. A computational linear elastic fracture mechanics-based model for alkali-silica reaction. Cement and Concrete Research, 2012, 42, pp.613-625. 10.1016/j.cemconres.2012.01.004 . hal-00701919

\section{HAL Id: hal-00701919 \\ https://hal-enpc.archives-ouvertes.fr/hal-00701919}

Submitted on 28 May 2012

HAL is a multi-disciplinary open access archive for the deposit and dissemination of scientific research documents, whether they are published or not. The documents may come from teaching and research institutions in France or abroad, or from public or private research centers.
L'archive ouverte pluridisciplinaire HAL, est destinée au dépôt et à la diffusion de documents scientifiques de niveau recherche, publiés ou non, émanant des établissements d'enseignement et de recherche français ou étrangers, des laboratoires publics ou privés. 


\title{
A computational linear elastic fracture mechanics-based model for alkali-silica reaction
}

\author{
Laurent Charpin ${ }^{*, a}$, Alain Ehrlacher ${ }^{\mathrm{a}}$ \\ ${ }^{a}$ Université Paris Est, UR Navier, École des Ponts ParisTech, 6-8 av Blaise Pascal, Cité Descartes, Champs-sur-Marne, 77455 \\ Marne-la-Vallée Cedex 2, France
}

\begin{abstract}
A fracture mechanics model for alkali-silica reaction (ASR) is presented that deals with the case of a concrete made up of dense spherical aggregates. Chemistry and diffusion (of ions and gel) are not modelled. The focus is put on the mechanical consequences of the progressive replacement of the aggregates by a less dense gel. A ring-shaped crack then appears in the cement paste depending on the pressure build-up, according to an incremental energy criterion. The stored elastic energy and deformation of each configuration are determined assuming that each aggregate is embedded in an infinite cement paste matrix, through Finite Element Analysis. We note a very different behaviour of aggregates of different sizes. Adding the contributions of different aggregates leads to an estimate of the free expansion of a concrete of given aggregate size distribution. Parameters of the model are identified, providing a good fit to experiments taken from Multon's work.
\end{abstract}

Key words: Alkali-aggregate reaction, Free expansion, Linear Elastic Fracture Mechanics, Energy criterion, Pessimum of aggregate size

\section{Introduction}

The alkali-silica reaction has been discovered in the 40's in the USA by Stanton [26]. It affects a very small fraction of concrete buildings, but it can be detrimental to the affected structures. First models were proposed in the 50's, and its study improved gradually when new experimental methods allowed to look inside the affected concrete more precisely. It has been observed that for the alkali-silica reaction to occur, three conditions need to be simultaneously verified: presence of reactive aggregates, high water content, and high alkali concentration. However, no consensus was reached on most parts of the reaction mechanisms. The alkali-silica reaction is visible through expansion and/or superficial cracking of macroscopic parts. Resistance to traction is much more affected than resistance to compression. The elasticity modulus decreases and plastic deformation increases. Microscopically a network of microcracks grows because of swelling of reactive sites where amorphous gels are created. One can sometime observe reaction rims and decohesion at the cement paste/aggregates interface.

These microcracks seem to play an important role in the macroscopic expansion of concrete structures. ASR is not always detrimental: when the gels find enough space to expand without cracking the cement paste, almost no macroscopic expansion is observed. Microcracks also play an important role concerning the anisotropy of the expansion when the concrete structure is loaded. Therefore in

\footnotetext{
* Corresponding author

Email address: laurent.charpin@enpc.fr (Laurent Charpin)
}

Preprint submitted to Elsevier this article, we will focus on the initiation and propagation of ASR microcracks, at the level of the reactive sites. First, we recall the main points of existing ASR models. We then explain briefly why we propose a new fracture model for ASR and its differences with existing ones. Then follow the description of the considered elementary volume and the computation of the energies used in the energy fracture criterion presented right after. The simple rule used to sum the contributions of different elementary volumes to macroscopic expansion comes next. Later, we explain which parameters influence the results of the model, focusing on aggregate size and the properties of the ASR gel. Finally, we discuss the potential of our model for reproducing experimental expansion curves.

\section{Mechanical modelling of ASR}

Amongst the mechanical models for ASR, the focus is placed on different physical phenomena according to the goal of the authors. For instance, Dormieux and Lemarchand $[15,14,6]$ have a microporomechanics approach. They compare the mechanical consequences of the topochemical and through-solution reaction mechanisms in [15]. It leads them to show that whether gelification occurs homogeneously in the porous space (through-solution mechanism), or mainly close to the sites of silica dissolution (topochemical mechanism), the usual S-shaped (sigmoid) expansion curve can be obtained. This expansion shape is recovered both if the gel is created in a crack family or homogeneously through the medium, but the characteristic expansion times differ. They also show that the 
compressibility of the gel might be important to consider. In $[14,6]$, they focus on the topochemical option and create the gel in a family of cracks representing the interface transition zone (ITZ) around aggregates. The effect of an external stress (isotropic or not) on the expansion is studied. They assume that the gel production stops when a crack is closed due to external stresses. This allows a good reproduction of Larive's tests [13] which show an anisotropy of swelling under anisotropic stresses, and the diminution of total swelling above a certain load.

Ichikawa's model $[12,11]$ is original because it takes into account the different role of different kind of precipitates according to their calcium content and hydration. This model is restricted to homogeneous and dense siliceous aggregates, in which the attack takes place gradually from the surface to the center. The expansion mechanism is related to reaction rim cracking, not paste cracking. Associated with a diffusion model for calcium and alkali ions, this fracture model reproduces aggregate fraction and size pessimum effects. They mention a thermodynamical evaluation of the expansion pressure that the gels can develop of $400 \mathrm{MPa}$.

The model of Suwito [28] considers the attack of a homogeneous and dense aggregate (crushed glass) by alkali hydroxides during the 14 days accelerated test ASTM C1260. It aims at reproducing the pessimum size effect. The model is divided into two parts: an application of the composite theory to characterise the expansion and internal pressure generated by ASR with aggregates of different sizes, and an application of diffusion theories to describe the chemical attack and the gel flow in the cement paste porosity. This problem is finally solved analytically using the unknown pressure at the surface of the aggregate as boundary condition. This formalism allows reproducing the aggregate pessimum size effect.

Sellier [24] proposed a model involving many physical phenomena and taking into account the probabilistic dispersion of various parameters. Diffusion in the porous space, cement paste cracking, capillary gel permeation are taken into account. The macroscopic swelling is estimated through the created crack volume. The model gives a good estimate of the pessimum aggregate volume fraction. In the same spirit as Capra in [5], the macroscopic part of the model reproduces the anisotropic swelling under anisotropic stresses, and the effect of the material heterogeneity.

The strictly mechanical model of Bažant [2] (as opposed to [1] which deals with other physical phenomena) aims at reproducing the pessimum effects when crushed recycling glass is used in concrete, in the ASTM C-1260 mortar bar accelerated test. The gel swelling is attributed to water imbibition. The cracking around spherical aggregates is calculated from an analytical stress intensity factor. The gel permeation in the connected porosity is taken into account. This procedure leads to a good reproduction of the pessimum size effect on expansion, and the calculated resistance in traction after ASR presents the same pessimum size as the measured compression resistance, but there is no quantitative agreement between predictions and measurements. Most parameters are obtained by curve fitting.

Multon gave a model [16] aiming at explaining the role of aggregate size and alkali content observed in two series of experiments [17], [16] and Poyet's work [21]. The volume of gel produced by each aggregate size is deduced from its volume fraction and alkali content. It is then corrected to take into account the escape of a part of the gel into the porosity of the surrounding cement paste. Finally, the average deformation is related linearly to the aggregate volume variation through a parameter to be identified, but no computation of fracture mechanisms is proposed. However the pessimum size behaviour is well reproduced. In a previous model [18], the damage of the cement paste surrounding the aggregate under attack reproduces the microcracking induced by the pressure build-up in the gel. Our model is from many points of view connected to this model, the main difference being that we consider explicit crack growth where they use damage.

Despite the number and variety of microscopic models for ASR, we thought that the description of cracking of the cement paste under ASR and its consequences could be improved. The models of Lemarchand and Dormieux $[15,14,6]$ are the closest to be able to reproduce the anisotropy of swelling effects, but we are interested in modelling cracking around aggregates, which they do with a geometry with which the way cracks grow is not satisfactory to us. Itchikawa $[12,11]$ deals with the probabilistic aspects of fracture in such a way that it seems too complicated to be extended to anisotropy. Suwito [28] considers swelling of aggregates and diffusion of gel, but no cracking occurs. Sellier [24] considers the cracks as penny-shaped, not taking into account the fact that they develop around aggregates. The approach of Bažant [2] is very interesting to us, but we are not fully satisfied with some assumptions such as the fact that the aggregates are placed on a periodical grid, and his estimation of the average deformation.

\section{Restrictions of the model proposed in this paper}

In this first attempt to understand the behaviour of an aggregate submitted to an attack, we only study a simplified mechanical problem related to ASR. Our goal is to understand the role of cracking of the cement paste in the swelling of concretes submitted to ASR. Therefore, we restrict our model to the simplest elements leading to the appearance of a crack in the cement paste under gel pressure. We want to understand how the sizes of aggregates influence cracking and hence, ASR expansion. The model follows many ideas of Bažant mechanical model for ASR [2]. Let us explain the main differences here. First, in our model, no assumption is made about the periodicity of the aggregates in the cement paste. Instead, each aggregate 
is assumed to be embedded in infinite cement paste. Second, the basis for crack propagation is a Finite Element Analysis (FEA), instead of an interpolation between analytically known stress intensity factors. On this point, our approach is close to that of Xiao [31] where the authors study, through FEA, the evolution of stress intensity factors for ring cracks surrounding inclusions when varying various parameters, except that we work directly on energies. We also compute the volume available to the gel by FEA which makes it simpler than what is done for example in [2] where it is deduced from the cracked medium's compliance which is obtained by integration (with respect to the crack size) of the stress intensity factor. Then, our determination of average deformations far from the reaction sites is quite simple. Finally we make no assumptions about the compressibility of the ASR products relatively to the cement paste and we briefly discuss its importance (like in [15]). Our goal in this article is to build a reliable method to create and propagate cracks that we'll be able to use under external loadings to study the anisotropy of expansion, later on. We plan to use this methodology to finally be able to extract macroscopic information about the anisotropy of swelling and decrease of material properties that can be used in structure-size FEA.

\section{Description of the behaviour of an elementary volume}

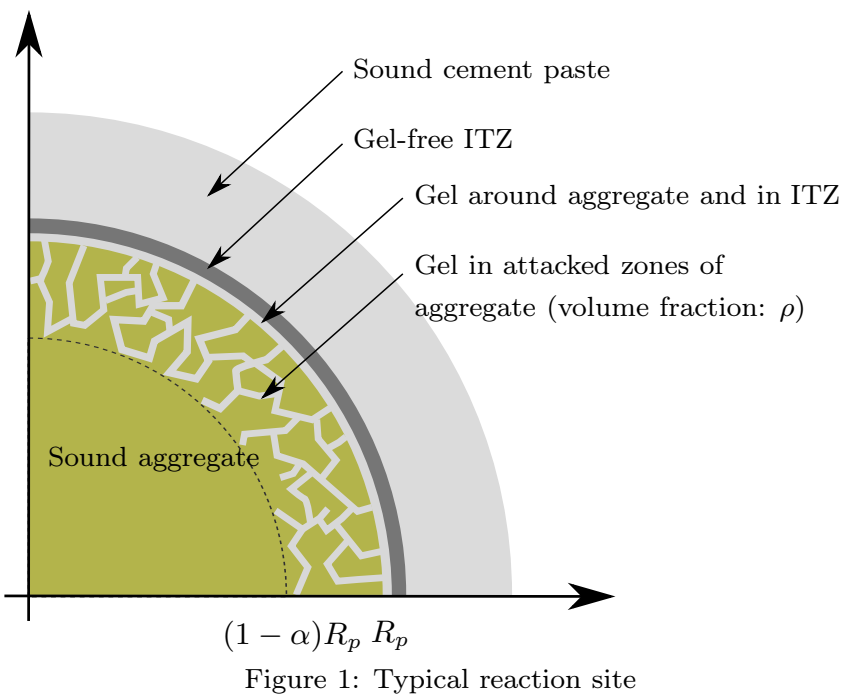

In this section we study in detail the behaviour of an aggregate surrounded by the infinite cement paste matrix, under stress-free outside boundary conditions (at infinity) (Fig. 1). The main assumptions and weaknesses of the model her are:

- the aggregates are spherical

- the attack occurs homogeneously from the surface of aggregates, but only a fraction $\rho$ of the aggre- gate is dissolved, which represents the volume fraction of reactive silica contained in the aggregate. This is an important assumption which could be completely wrong for some aggregates which contain pockets rather than homogeneously spread reactive silica. This choice is related to the decision to model the behavior of rapidly reacting aggregates, as those tested for example by Giaccio [10] in his two first concrete mixes: a highly reactive siliceous orthoquartzite and a natural sand containing volcanic glass. For these two types of aggregates, the reaction mostly takes place at the surface of the aggregates, inducing a pressure build-up and crack propagation into the cement paste. Ponce also classifies aggregates as rapid-reacting and slow-reacting [20]. He explains the mechanism of dissolution of the reactive siliceous phase (opal, chalcedony) in an orthoquartzite aggregate where the reactive silica plays the role of a cement for less reactive part of aggregates (quartz grains). Hence, when it is dissolved, the aggregates looses its cohesion with the cement paste. The case of slow-reactive aggregates, where reactive phases are usually embedded in non reactive ones inside the aggregate, is completely different. Some examples are given in the two just cited works [20] and [10] and a detailed description of the expansion mechanism for such aggregates is given in Ben Haha's work [4] and [3]. Dunant [7] and [8] has built a numerical model for alkali silica reaction where the reactive zones are placed inside the aggregates. Their expansion induced damage in the aggregate. Reinhardt [22] has built a model for ASR which is focused on the fracture of aggregates under the internal pressure of the gel.

- the first layer of cement paste around the aggregate represents the Interface Transition Zone (ITZ), of thickness $l_{c}$ (order of magnitude: $1 \mu \mathrm{m}$ ), which is a zone of large porosity compared to the rest of the cement paste. Therefore, we assume that the produced gel doesn't penetrate in the cement paste porosity, except for the interface transition zone which is assumed to be of porosity 1 . However, since we think that some pressure is required for the gel to invade the ITZ's porosity, the filling of this zone will be assumed to increase with pressure, until it is full. The presence of this zone of higher porosity and reduced mechanical properties, where the gel concentrates, supports the idea that as far as expansion is concerned and once attack has begun, everything is as if the aggregate was unbounded from the cement paste.

- the aggregates don't see each other mechanically: cracking happens as if each of them was embedded in an infinite cement paste matrix. This assumption is highly disputable, due to the high aggregate concentration of concretes (half of the total volume is 
common). We intend to improve the micromechanical description of the concrete sample in a further article in order to better take into account the large aggregate concentration and estimate the reduction of macroscopic elastic properties.

We call $R_{p}$ the radius of the cavity in the cement paste. It is also the radius of the aggregate before the attack. The aggregate is chemically attacked in such a way that a fraction $\rho$ of its volume is replaced by a gel, starting from the outside. The degree of attack is described by the parameter $\alpha \in[0 ; 1]$. The undeformed radius of the sound part of the aggregate (that is if the pressure is released) at the degree of attack $\alpha$ is $R_{g}(\alpha)=(1-\alpha) R_{p}$. The total undeformed remaining volume of aggregate is the sum of the volume of sound aggregate plus the remaining volume in the attacked parts, representing a volume fraction of $(1-\rho)$ relatively to the original aggregate. Therefore it writes:

$$
V_{a 0}(\alpha)=\frac{4}{3} \pi R_{p}^{3}\left[(1-\alpha)^{3}+(1-\rho)\left[1-(1-\alpha)^{3}\right]\right]
$$

The aggregate and cement paste are considered linear isotropic elastic of Young's modulus and Poisson's ratio $\left(E_{a}, \nu_{a}\right)$ and $\left(E_{c}, \nu_{c}\right)$. The gel bulk modulus is $K_{g e l}$.

If we assume that the chemical reaction is expansive and the products occupy a volume $\delta$ times bigger than the aggregate, the volume of gel at the reaction extent $\alpha$, under zero pressure, is:

$$
V_{0}(\alpha)=\frac{4}{3} \pi \delta \rho R_{p}^{3}\left[1-(1-\alpha)^{3}\right]
$$

Let us stress that $\delta$ could be an effective value of the ratio of the gel volume to the aggregate volume accounting for physical phenomena such as the water imbibition by the gel, which is not explicitly described here. To keep our model simple, we consider it as a constant. We also assume that the gel is at constant pressure (whether in the aggregate, at the surface, or later in the ITZ and cracks), since ASR is a slow process. We study the cracking of the cement paste under the increasing pressure created by gel accumulation around the aggregate. Quantities relative to the gel, the aggregates, and the cement paste, respectively have the indexes $g e l, a$, and $c$.

\subsection{Study of the situation right after the appearance of an axisymmetric crack}

We suppose that the crack breaks the spherical symmetry of the system but keeps a cylindrical symmetry. The cement paste is cracked by a penny-shaped crack in the plane $\left(0, \underline{e}_{x}, \underline{e}_{y}\right)$. The crack is concentric with the aggregate (Fig. 2).

We assume that when in the uncracked state the pressure reaches a critical value (yet unknown), a crack of finite length is instantaneously created. Hence, the crack inside radius is $R_{p}$ and its outside radius is $(1+x) R_{p}$ in undeformed configuration. $x$ is a dimensionless parameter

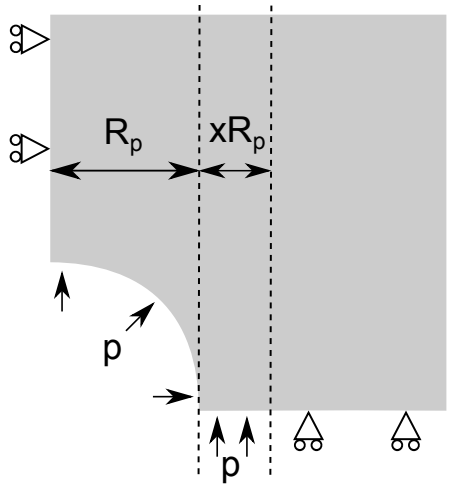

Figure 2: Crack considered

representing the size of the crack. The area of the created crack is:

$$
S(x)=\pi R_{p}^{2}\left[(1+x)^{2}-1\right]
$$

\subsubsection{Gel pressure}

This instantaneous crack creation leads to an increase of the volume accessible to the compressible gel and hence, a decrease of its pressure. We write the gel pressure $P(\alpha, x)$, to make explicit its dependence on the attack degree and crack size, keeping implicit other dependencies (on material and geometrical fixed parameters). We will use the same notation for volumes and energies.

Point of view of the gel

These quantities are related through the state law of the gel:

$$
V_{g e l}(\alpha, x)=V_{0}(\alpha)\left[1-\frac{P(\alpha, x)}{K_{g e l}}\right]
$$

Point of view of the solid

In this cracked configuration, no analytical expression is available to compute the volume variation of the cavity depending on the length of the crack, pressure, ... However, we can use dimensional analysis to have as few different cases to compute through finite elements analysis (FEA) as possible.

Let us consider the simple problem explained on Fig. 2 where the cracked cement paste is submitted to a pressure $P$ on the cavity surface and the crack lips. We are interested in the volume change of the cavity when pressure is applied. It shall depend on the pressure $P$, the cavity radius $R_{p}$, the relative crack size $x$ and the Young's modulus and Poisson's ration of the cement paste $\left(E_{c}, \nu_{c}\right)$.

First, due to the linearity of this elasticity problem, the volume variation of the cavity is proportional to the loading pressure $P$. Second, dimensional analysis allows us to write the volume variation in the form $\Delta V_{c}=R_{p}^{3} f\left(\frac{P}{E_{c}}\right) g\left(x, \nu_{c}\right)$. Where the functions $f$ and $g$ are dimensionless. Recalling that it is proportional to the loading pressure, we get $\Delta V_{c}=R_{p}^{3} \frac{P}{E_{c}} g\left(x, \nu_{c}\right)$.

For convenience of the expression, we write it in the following way, where $\alpha$ and $x$ are the two variables describing the system: 


$$
\Delta V_{c}(\alpha, x)=\frac{4}{3} \pi R_{p}^{3} \frac{P(\alpha, x)}{E_{c}} \Delta v(x)
$$

Where $\frac{4}{3} \pi \Delta v(x)$ is the volume change when a pore of unit radius surrounded by a crack of dimensionless length $x$ in a material of unit young modulus and Poisson ration $\nu_{c}$ is loaded by a unit pressure. Strictly speaking, $\Delta v$ depends on $\nu_{c}$ but we don't write it explicitly since it is easy to fix $\nu_{c}$ once and for all and to perform the calculations for this value. This function is know by FEA, by relating it to the stored elastic energy in the medium, as explained in $\S 4.1 .2$.

When there is no crack, it is easy to write a closed form expression of the dimensionless volume variation of the cavity:

$$
\Delta v(0)=\frac{3}{2}\left(1+\nu_{c}\right)
$$

The volume accessible to the gel can then be written as the sum of four terms:

- first, the volume left free by the aggregate erosion:

$$
\frac{4}{3} \pi R_{p}^{3} \rho\left[1-(1-\alpha)^{3}\right]
$$

- second, the volume corresponding to the hydrostatic compression of the sound part of the aggregate and the remaining proportion $(1-\rho)$ in the attacked zone (see Eq. 1), easily written using the bulk modulus of the aggregate $K_{a}=\frac{E_{a}}{3\left(1-2 \nu_{a}\right)}$ :

$$
\frac{P(\alpha, x)}{K_{a}} V_{a 0}(\alpha)
$$

- the contribution of the cement paste explained above, equal to $\Delta V_{c}(\alpha, x)$ (see Eq. 5)

- the volume of gel which could permeate into the ITZ, which we choose to depend linearly on the pressure until a threshold pressure $P_{0}$ is reached (when the ITZ is full) and to be proportional to the external surface of the considered aggregate:

$$
V^{I T Z}=4 \pi R_{p}^{2} l_{c} \min \left(1, \frac{P}{P_{0}}\right)
$$

Adding these contributions (Eqs. 7, 8, 5, 9):

$$
\begin{aligned}
& V_{\text {gel }}(\alpha, x)= \frac{4}{3} \pi R_{p}^{3}\left\{\rho\left[1-(1-\alpha)^{3}\right]\right. \\
&+\frac{P(\alpha, x)}{K_{a}}\left[(1-\alpha)^{3}+(1-\rho)\left[1-(1-\alpha)^{3}\right]\right] \\
&\left.+\frac{P(\alpha, x)}{E_{c}} \Delta v(x)+\frac{3}{R_{p}} l_{c} \min \left(1, \frac{P}{P_{0}}\right)\right\}
\end{aligned}
$$

And finally the pressure right after cracking is determined by solving the piecewise linear equation in $P(\alpha, x)$ obtained by saying that our two expressions for the volume of gel $V_{\text {gel }}$ (Eqs. 4 and 10) are equal (again using the aggregate bulk modulus $\left.K_{a}=\frac{E_{a}}{3\left(1-2 \nu_{a}\right)}\right)$ :

$$
\begin{aligned}
& \text { If } P>P_{0}, P(\alpha, x)= \\
& \frac{(\delta-1) \rho\left[1-(1-\alpha)^{3}\right]-\frac{3 l_{c}}{R_{p}}}{\frac{\delta \rho\left[1-(1-\alpha)^{3}\right]}{K_{g e l}}+\frac{\Delta v(x)}{E_{c}}+\frac{(1-\alpha)^{3}+(1-\rho)\left[1-(1-\alpha)^{3}\right]}{K_{a}}} \\
& \text { If } P<P_{0}, P(\alpha, x)= \\
& \frac{(\delta-1) \rho\left[1-(1-\alpha)^{3}\right]}{\frac{\delta \rho\left[1-(1-\alpha)^{3}\right]}{K_{g e l}}+\frac{\Delta v(x)}{E_{c}}+\frac{(1-\alpha)^{3}+(1-\rho)\left[1-(1-\alpha)^{3}\right]}{K_{a}}+\frac{3 l_{c}}{P_{0} R_{p}}}
\end{aligned}
$$

These two expressions are identical for $P=P_{0}$. Therefore, switching from Eq. 12 to Eq. 11 leads to no discontinuity of pressure with respects to the variations of the attack degree $\alpha$. And we define the dimensionless pressure $\bar{p}(\alpha, x)=\frac{P(\alpha, x)}{E_{c}}$. The dependence in $R_{p}$ passes through the presence of $x$ which depends on $R_{p}$ and is explicit in the term related to the ITZ.

\subsubsection{Stored elastic energy}

The stored elastic energies in the aggregate and gel are computed in closed form:

Elastic energy stored in the gel

$$
\begin{aligned}
E_{g e l}^{e l}(\alpha, x) & =\frac{1}{2} \frac{P^{2}(\alpha, x)}{K_{g e l}} V_{0}(\alpha) \\
& =\frac{2 \pi}{3} \frac{\rho \delta R_{p}^{3}}{K_{g e l}}\left[1-(1-\alpha)^{3}\right] P^{2}(\alpha, x)
\end{aligned}
$$

Elastic energy stored in the aggregate

$$
\begin{aligned}
& E_{a}^{e l}(\alpha, x)= \\
& 2 \pi R_{p}^{3}\left[(1-\alpha)^{3}+(1-\rho)\left[1-(1-\alpha)^{3}\right]\right] \frac{1-2 \nu_{a}}{E_{a}} P^{2}(\alpha, x)
\end{aligned}
$$

Elastic energy stored in the cement paste

For the cement paste matrix, no closed form expression is available. We use the same approach as for the volume variation of the cavity in cracked cement paste. We are interested in the elastic energy stored in the cement paste under pressure $P$. It shall depend on $P, R_{p}, x$, and $\left(E_{c}, \nu_{c}\right)$. Dimensional analysis leads to write the elastic energy in the following form: $E_{c}^{e l}=R_{p}^{3} \frac{P^{a}}{E_{c}^{a-1}} g\left(x, \nu_{c}\right)$ where $a \in \mathbb{R}$ and the function $g$ is dimensionless. Then due to the linearity of the problem, the deformation and stress tensors are proportional to the loading pressure $P$. The elastic energy, which is the integration of the contraction of those tensors over the full domain is finally proportional to $P^{2}$, that is $a=2$. Finally we write it in the following manner:

$$
E_{c}^{e l}(\alpha, x)=2 \pi R_{p}^{3} \frac{P^{2}(\alpha, x)}{E_{c}} e(x)
$$


Where $2 \pi e(x)$ represents the stored elastic energy under unit pressure, unit cavity radius, and a unit Young's modulus of the material. Again, the dependence on $\nu_{c}$ is not written explicitly. We also have the analytical expression of this quantity when there is no crack:

$$
e(0)=\frac{1+\nu_{c}}{2}
$$

And the numerical values of this function for $x>0$ are determined using FEA (Fig. 3). At the same time the numerical values for $\Delta v(x)$ are known thanks the equality of the stored elastic energy and work of the pressure:

$$
E_{c}^{e l}(\alpha, x)=\frac{1}{2} P(\alpha, x) \Delta V_{c}(\alpha, x)
$$

Hence,

$$
\Delta v(x)=3 e(x)
$$

We see on this curve that there is a horizontal tangent in

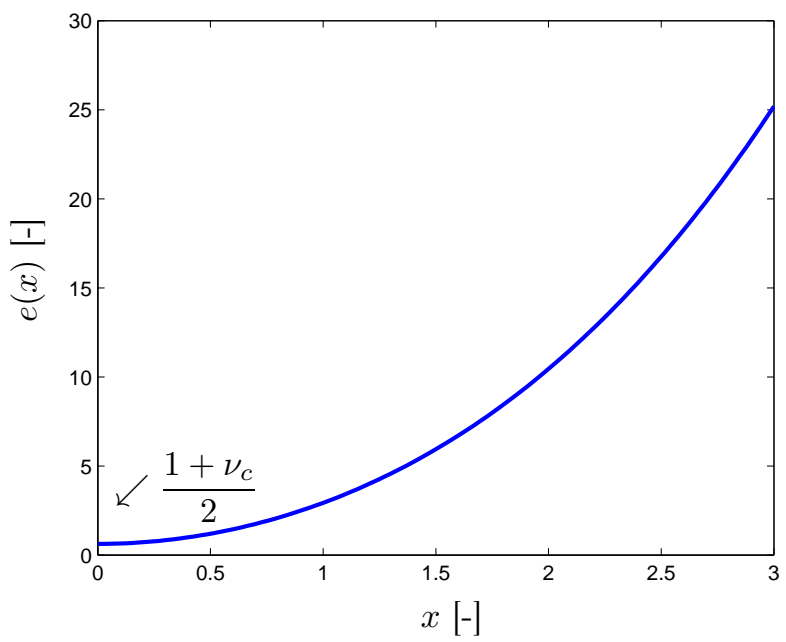

Figure 3: FE estimate of the stored elastic energy.

$x=0: \frac{\partial e(x)}{\partial x}(x) \stackrel{x \rightarrow 0}{\longrightarrow} 0$

Total elastic energy in the cracked configuration

$$
\begin{aligned}
& E_{\text {total }}^{e l}(x, \alpha)=2 \pi R_{p}^{3}\left[\frac{\delta \rho\left(1-(1-\alpha)^{3}\right)}{3 K_{\text {gel }}}+e(x)\right. \\
& \left.+\left[(1-\alpha)^{3}+(1-\rho)\left(1-(1-\alpha)^{3}\right)\right] \frac{1-2 \nu_{a}}{E_{a}}\right] P^{2}(\alpha, x)
\end{aligned}
$$

\section{Thermodynamical study of the possible instan- taneous initiation of a crack of finite length. Crack propagation. Energy criterion}

\subsection{Energy balance}

The creation of the crack requires a certain amount of energy that is supplied by the release of elastic energy. This released energy is simply written:

$$
E^{R e l}(\alpha, x)=E_{\text {total }}^{e l}(\alpha, 0)-E_{\text {total }}^{e l}(\alpha, x)
$$

Using Eq. 19, we can see that the released energy $E^{R e l}(\alpha, x)$ is proportional to $R_{p}^{3}$, that is $E^{\operatorname{Rel}}(\alpha, x)=\pi R_{p}^{3} e^{\operatorname{Rel}}(\alpha, x)$ where $e^{R e l}(\alpha, x)$ is independent of $R_{p}$.

$$
\begin{gathered}
e^{R e l}(\alpha, x)=2\left[( P ^ { 2 } ( \alpha , 0 ) - P ^ { 2 } ( \alpha , x ) ) \left(\frac{\delta \rho\left(1-(1-\alpha)^{3}\right)}{3 K_{\text {gel }}}\right.\right. \\
\left.+\frac{\left[(1-\alpha)^{3}+(1-\rho)\left(1-(1-\alpha)^{3}\right)\right]\left(1-2 \nu_{a}\right)}{E_{a}}\right) \\
\left.+\frac{1+\nu_{c}}{2} P^{2}(\alpha, 0)-e(x) P^{2}(\alpha, x)\right]
\end{gathered}
$$

Assuming that the energy required to create a crack $E^{\text {diss }}(x)$ is proportional to the crack surface, we realize it is proportional to $R_{p}^{2}$, so it can be written $E^{d i s s}(x)=\pi R_{p}^{2} e^{\text {diss }}(x)$ where $e^{\text {diss }}(x)$ is independent on $R_{p}$ and writes:

$$
e^{\text {diss }}(x)=G_{c}\left[(1+x)^{2}-1\right]
$$

For the creation of a crack to be possible at the degree of attack $\alpha$, it is necessary that:

$$
\exists x \in] 0,+\infty\left[, E^{R e l}(\alpha, x) \geq E^{d i s s}(x)\right.
$$

We consider this necessary condition as sufficient and therefore, we use the equality of released energy and dissipated energy as a crack initiation criterion. Now that we know how to obtain the expressions for all the energies needed, lets discuss the shape of these curves to understand better the conditions for cracking (Fig. 4 and 5). The analytical expression of $E^{\text {diss }}$ is known (Eq. 22). $E^{R e l}(x)$ has the following limits at constant $\alpha$ :

- $E^{R e l}(\alpha, x) \stackrel{x \rightarrow 0}{\longrightarrow} 0$ because if no crack appears at constant $\alpha$, there is no energy release

- $\frac{\partial E^{R e l}}{\partial x}(\alpha, x) \stackrel{x \rightarrow 0}{\longrightarrow} 0$ because it is proportional to $\frac{\partial e(x)}{\partial x}(x)$ (see Eqs. 21, 18, 11, and 12) which has a horizontal asymptote in $x=0$ (see Fig. 3 )

- $E^{R e l}(\alpha, x) \stackrel{x \rightarrow+\infty}{\longrightarrow} E_{\text {total }}^{e l}(\alpha, 0)$ and $\frac{\partial E^{R e l}}{\partial x}(\alpha, x) \stackrel{x \rightarrow+\infty}{\longrightarrow} 0$ because the stored elastic energy in a configuration with a crack of infinite size is zero.

At constant $x, E^{R e l}$ increases with increasing $\alpha$. We display the shape of $E^{R e l}(x)$ for different values of $\alpha$ (see Fig. 4).

Let us explain the initiation of a crack:

- Originally, $\alpha=0$ so $E^{R e l}=0$

- The attack begins, so $\alpha$ starts increasing. Hence, $E_{\text {total }}^{e l}(\alpha, 0)>0$ and $E^{R e l}(\alpha, x)>0$. However, since $\frac{d E^{\text {diss }}}{d x}(x=0)>0$ while $\frac{\partial E^{R e l}}{\partial x}(\alpha, x=0)=0$, at the beginning of attack we have $\forall x, E^{\text {Rel }}(\alpha, x)<$ $E^{\text {diss }}(x)$

- From now on, since we decided to use an energy criterion for crack initiation, a crack of length $x_{c}$ will 


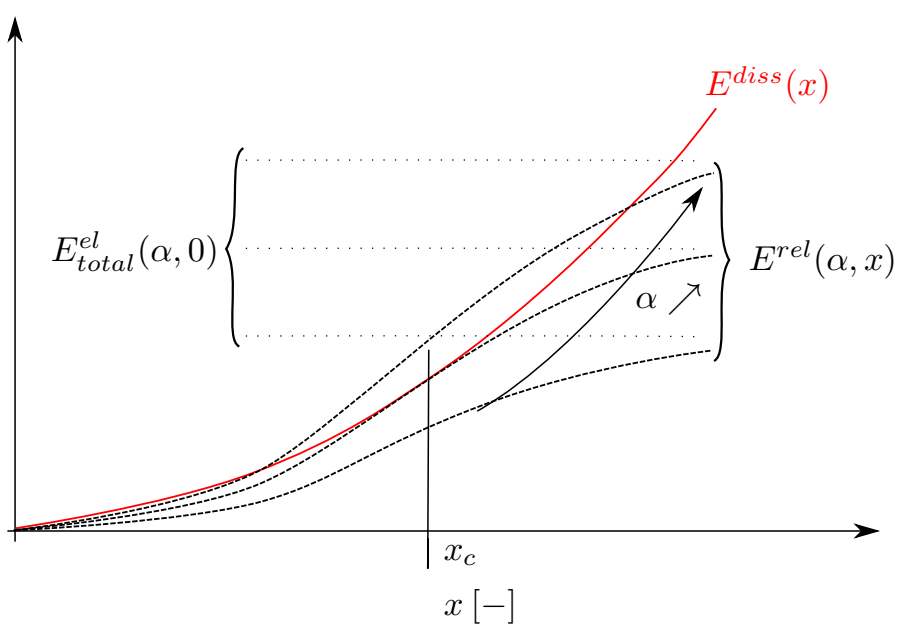

Figure 4: Dissipated and released energies when a crack of length $x$ is created

be created as soon as we reach a critical degree of attack $\alpha$ such as:

$$
\exists x \mid E^{R e l}(\alpha, x)=E^{d i s s}(x)
$$

Since $\alpha \leq 1$, this may never happen. In this case even at full attack, no crack will be created. We discuss this in $\S 5.2$. Moreover, since the loading increases continuously, the first attack degree leading to the creation of a crack of length $x_{c}$ is also characterized by the fact that $E^{R e l}$ and $E^{\text {diss }}$ are tangent at this point, that is for a given initial aggregate radius $R_{p}$, the solution $\left(\alpha_{c}\left(R_{p}\right), x_{c}\left(R_{p}\right)\right)$ is fully characterized by:

$$
\left\{\begin{array}{l}
E^{R e l}\left(\alpha_{c}\left(R_{p}\right), x_{c}\left(R_{p}\right)\right)=E^{\text {diss }}\left(x_{c}\left(R_{p}\right)\right) \\
\frac{\partial E^{\text {Rel }}}{\partial x}\left(\alpha_{c}\left(R_{p}\right), x_{c}\left(R_{p}\right)\right)=\frac{d E^{\text {diss }}}{d x}\left(x_{c}\left(R_{p}\right)\right)
\end{array}\right.
$$

These two conditions translate in terms of energy rates by the equality of the two grey surfaces on Fig. 5.

- Once the crack has been initiated, it is important to study how it evolves, since the final goal is to mix different sizes of aggregates which will crack their surrounding cement paste at different times. We assume that the existing crack doesn't modify the spherical symmetry of the attack of the grain. Therefore, this attack is still fully described by the parameter $\alpha$. Starting from $\left(\alpha_{c}, x_{c}\right)$, the propagation of the crack when $\alpha$ further increases is governed by the energy rate equation $\frac{\partial E^{R e l}}{\partial x}(\alpha, x)=\frac{d E^{d i s s}}{d x}(x)$. Since $\frac{\partial^{2} E^{R e l}}{\partial x^{2}}(\alpha, x)<0$ and $\frac{d^{2} E^{d i s s}}{d x^{2}}\left(x_{c}\right)>0$ around the current point $(\alpha, x)$ (see Fig. 5), and $\frac{\partial E^{R e l}}{\partial x}(\alpha, x)$ is continuous in $\alpha, x$ evolves continuously with $\alpha$ when the latter further increases. Hence, the propagation is stable.

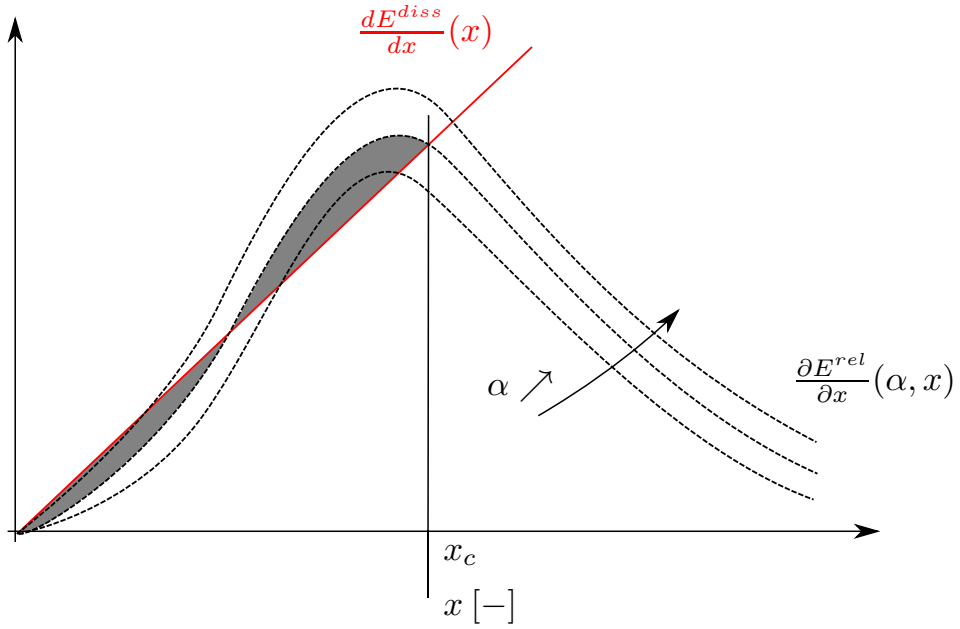

Figure 5: Dissipated and released energies rates when a crack of length $x$ is created

\subsection{Smallest aggregate radius that can lead to the failure} of the cement paste

All other parameters fixed $\left(K_{g e l}, \delta, l_{c}, P_{0}, \rho,\left(E_{c}, \nu_{c}\right)\right.$, $\left.\left(E_{a}, \nu_{a}\right)\right)$, since $E^{\text {diss }}(x)=\pi R_{p}^{2} e^{d i s s}(x)$ and $E^{\text {Rel }}(\alpha, x)=$ $\pi R_{p}^{3} e^{R e l}(\alpha, x)$ (where $e^{\text {diss }}$ and $e^{R e l}$ are independent of $R_{p}$ ), and at given attack degree $\alpha$, the aggregates sizes for which cracking is possible are such as $\exists x \mid E^{\text {diss }}(x) \leq$ $E^{R e l}(\alpha, x)$, or equivalently $\exists x \mid \frac{e^{d i s s}(x)}{e^{\text {Rel }}(\alpha, x)} \leq R_{p}$. Therefore, at given $\alpha$ the smallest aggregate size for which cracking is possible is:

$$
R_{p}=\inf _{x} \frac{e^{d i s s}(x)}{e^{R e l}(\alpha, x)}=\inf _{x} Q(\alpha, x)
$$

Therefore, there exists an aggregate radius $R_{\text {inf }}$ below which cracking is impossible which means that the pressure created by the full attack of the aggregate is not high enough to crack the cement paste matrix. Below this radius $R_{\text {inf }}$,

$$
\forall x, E^{R e l}(1, x) \leq E^{\text {diss }}(x)
$$

Hence the smallest radius for which the cement paste will be cracked before full attack depends on $\frac{G_{c}}{E_{c}}, \frac{E_{c}}{K_{g e l}}, \nu_{c}, \delta$, $l_{c}, P_{0}, \rho, \frac{E_{c}}{E_{a}}$, and $\nu_{a}$.

We can plot the ratio $Q(1, x)=\frac{e^{d i s s}(x)}{e^{\operatorname{Rel}}(1, x)}$ which is used to determine $R_{\text {inf }}$ (Fig. 6), in the simplifying case where $\rho=1, l_{c}=0$ (in this case $P_{0}$ has no influence). We also obtain the corresponding relative crack size $x_{i n f}$ (which is not the smallest possible crack size, but the crack size corresponding to $R_{\text {inf }}$ ). We take $E_{c}=20 \mathrm{GPa}, E_{g}=60 \mathrm{GPa}$, $\nu_{c}=\nu_{g}=0.25, G_{c}=40 \mathrm{~J} . \mathrm{m}^{-2}, K_{g e l}=1 \mathrm{GPa}$, and $\delta=1.03$. The Poisson's ratios and Young's modulus are classical for cement paste and aggregates, the fracture energy is taken from Wittmann [30], and the two last parameters are chosen arbitrarily, since they are not well-known, which will be discussed later on.

Now, starting from the knowledge of the volume fraction of each aggregate size at the macroscopic level and 


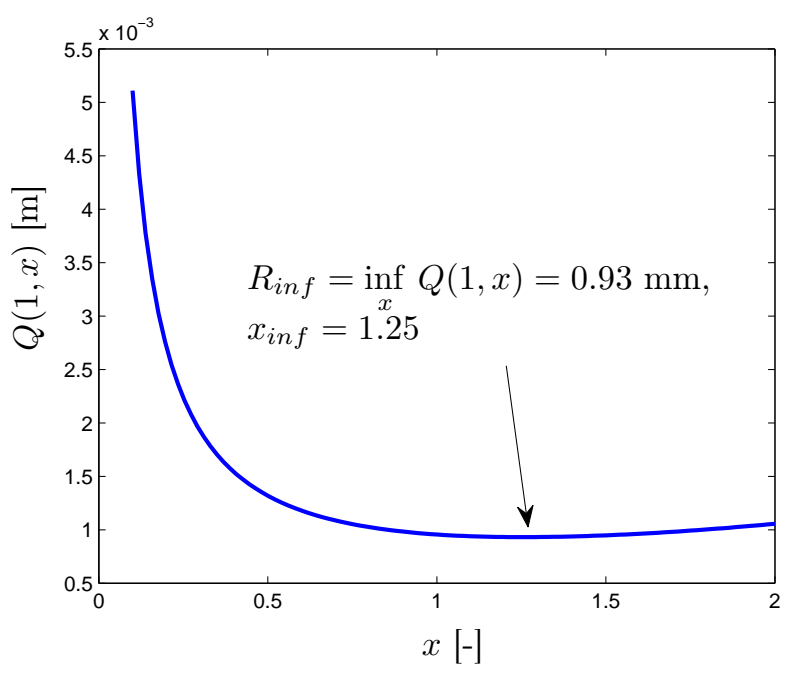

Figure 6: The ratio we minimize to find $\left(R_{\text {inf }}, x_{i n f}\right): Q(1, x)=$ $\frac{e^{d i s s}(x)}{e^{R e l}(1, x)}$

that of the pressure and the crack size for each aggregate size at the microscopic level, we need to define a macroscopic deformation.

\section{Study of the macroscopic deformation}

First we consider one aggregate in infinite cement paste and a domain $\Omega$ including the aggregate. Its current state is described by the couple $(x, P(\alpha, x))$. Let us consider $\underline{\underline{U}}=\int_{\partial \Omega}(\underline{u} \otimes \underline{n})^{s} d S$ where $\underline{u}$ is the displacement field, $\underline{n}$ the outward normal to $\Omega, \partial \Omega$ is the domains border, and $s$ is used to take the symmetric part of the tensor. TakanaMori's theorem [29] states that if $\Omega$ is a sphere, $\underline{U}$ does not depend on its size and position as long as it includes the cavity. Suppose there is only one aggregate size $R_{p}$ representing a volume fraction $f$ throughout the concrete. Then the sphere $\Omega$ has to be chosen such that $f=\frac{\frac{4}{3} \pi R_{p}^{3}}{|\Omega|}$ to make sure that the volume fraction of the aggregate in the domain used to compute the average deformation due to the attack is equal to the volume fraction in the concrete considered. Finally we define the macroscopic deformation $\underline{\underline{E}}=\frac{1}{|\Omega|} \underline{\underline{U}}=\frac{f}{\frac{4}{3} \pi R_{p}^{3}} \underline{\underline{U}}$.

Then, we need to take into account the aggregate size distribution. To each aggregate size $R_{p, i}$ corresponds a volume fraction $f_{i}$ in the concrete. Each aggregate class contributes to the macroscopic deformation through a partial average deformation defined as:

$$
\underline{\underline{E}}_{i}=\frac{f_{i}}{\frac{4}{3} \pi R_{p, i}^{3}} \underline{\underline{U}}_{i}
$$

where $\underline{\underline{U}}_{i}$ is computed relatively to the aggregate of size $R_{p, i}$, a crack of size $x_{i}$, and a pressure $P_{i}=P\left(\alpha_{i}, x_{i}\right)$. The macroscopic deformation is then defined as:

$$
\underline{\underline{E}}=\sum_{\substack{\text { aggregate } \\ \text { size } i}} \underline{E}_{i}
$$

A corollary of Takana-Mori's theorem [29] for which we will give our own proof in a later paper relates the volumetric part of $\underline{\underline{U}}_{i}$ to the dimensionless stored elastic energy in the cement paste surrounding the considered aggregate (defined in Eq. 15) through the expression:

$$
\operatorname{tr} \underline{\underline{U}}_{i}=\frac{R_{p, i}^{3} P\left(\alpha_{i}, x_{i}\right)}{E_{c}} \frac{4 \pi}{3} \frac{1+\nu_{c}}{1-\nu_{c}}\left[1-2 \nu_{c}+e\left(x_{i}\right)\right]
$$

Eq. 30 finally gives us access to the macroscopic volumetric deformation:

$$
\underline{\underline{E}}=\sum_{\substack{\text { aggregate } \\ \text { size } i}} f_{i} \frac{P\left(\alpha_{i}, x_{i}\right)}{E_{c}} \frac{1+\nu_{c}}{1-\nu_{c}}\left[1-2 \nu_{c}+e\left(x_{i}\right)\right]
$$

\section{Numerical results: effect of aggregate size on crack initiation and volume changes}

In this section, we strictly study the behaviour of the fracture model. To focus on this part of our work, we set $l_{c}=0$ and $\rho=1$. As soon as the gel is created, a significant pressure build-up is observed. We want to apply our model to see if it allows explaining the expansions observed in ASR. To do so, we need some reliable values of the different parameters involved. Recalling that $x_{i n f}$ depends on $\frac{E_{c}}{K_{g e l}}$, $\nu_{c}, \delta, \frac{E_{c}}{E_{a}}, \nu_{a}$ and $R_{\text {inf }}$ depends on theses quantities plus $\frac{G_{c}}{E_{c}}$, we are going to study the influence of the aggregate radius $R_{p}$, the ratio of gel to aggregate volume $\delta$ and the gel compressibility $K_{\text {gel }}$ on first cracking and crack length at full attack. The other parameters which vary less or are easier to determine for a given concrete will be set to the values of $E_{c}=20 \mathrm{GPa}, E_{g}=60 \mathrm{GPa}, \nu_{c}=\nu_{g}=0.25$, $G_{c}=40 \mathrm{~J} . \mathrm{m}^{-2}$.

\subsection{Study of the first and final cracking}

\subsubsection{Smallest aggregate size that can lead to the fracture of the cement paste: $R_{\text {inf }}$}

Let us first study how $R_{\text {inf }}$ varies when changing $\delta$ and $K_{g e l}$. Is is important to study this behaviour, because the swelling of the gel and its compressibility are not well known. In 2005, Phair claimed to publish the first measurements of ASR gel bulk modulus [19]. The measured values are of the order of $10 \mathrm{GPa}$. His experimental setup seems to measure the undrained bulk modulus of the gel. The gel is porous, so its drained modulus might be very different, and it seems that since gel creation is very slow, water movements have time to occur during the pressure build-up. However the instant of cracking might be very short. Hence, it is possible that the effective bulk modulus of the gel during cracking is closer to high values such as reported by Phair. Moreover, the different sorts of gel that are produced in different chemical environments might also have very different and time-dependant values of these parameters as observed on the storage and loss moduli of synthetic alkaline-calcium silica gels by Gaboriaud [9]. Since the swelling of the gel is often explained 
by water absorption, they might also differ significantly according to the saturation degree of the concrete. Hence, in a predictive model, they would probably have to be determined by an optimization procedure.

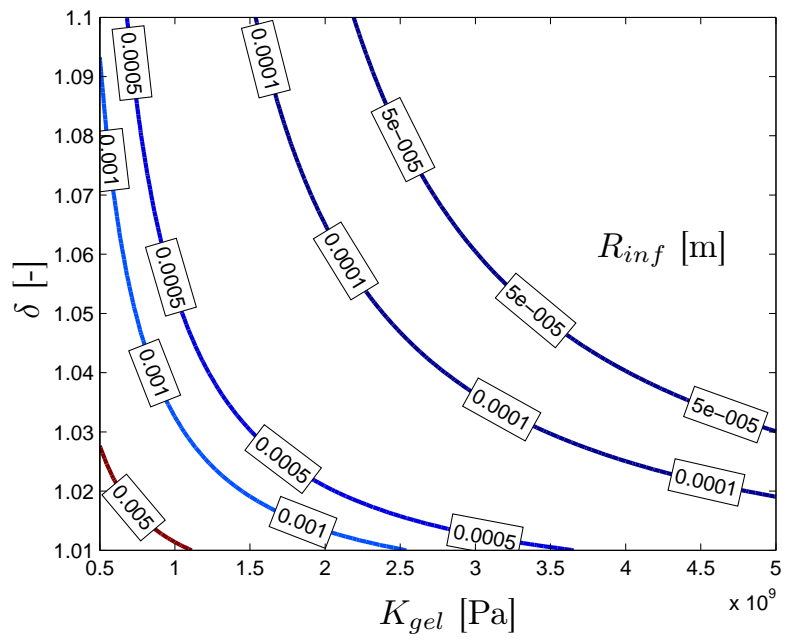

Figure 7: Minimum radius to crack the cement paste: $R_{i n f}\left(\delta, K_{g e l}\right)$

We can see on Fig. 7 (which is build using Eq. 26 for various values of $K_{\text {gel }}$ and $\delta$ ) that increasing the coefficient of expansion $\delta$ or the compressibility $K_{\text {gel }}$ decreases the radius of the smallest aggregate that leads to fracture initiation. When $\delta \longrightarrow 1$, cracking becomes impossible, as well as when the gel is too soft $\left(K_{\text {gel }} \longrightarrow 0\right)$.

\subsubsection{First and final crack sizes varying $R_{p}, K_{\text {gel }}$, and $\delta$}

These three parameters are very important to correctly determine the crack initiation and propagation around an aggregate as can be seen on Fig. 8, 9, 10 where we represent the first $\left(x_{c}\right)$ and final $\left(x_{\max }\right)$ crack size when changing these parameters. The final crack size $x_{\max }$ is defined as the crack size $x$ at full attack of the aggregate $\alpha=1$. The two unchanged parameters (out of 3 ) in each case have the values $K_{\text {gel }}=1 \mathrm{GPa}, \delta=1.03$ and $R_{p}=1.5 \mathrm{~mm}$.

A similar behaviour observed varying $R_{p}, K_{g e l}$, or $\delta$ (Fig. 8, 9, 10): for small values, cracking is impossible even at full attack $(\alpha=1)$ then, cracking becomes possible. The initial crack size $\left(x_{c}\right.$ as defined by Eq. 25) further decreases, and the final crack size $\left(x_{\max }\right)$ increases when increasing $\delta, K_{\text {gel }}$ or $R_{p}$.

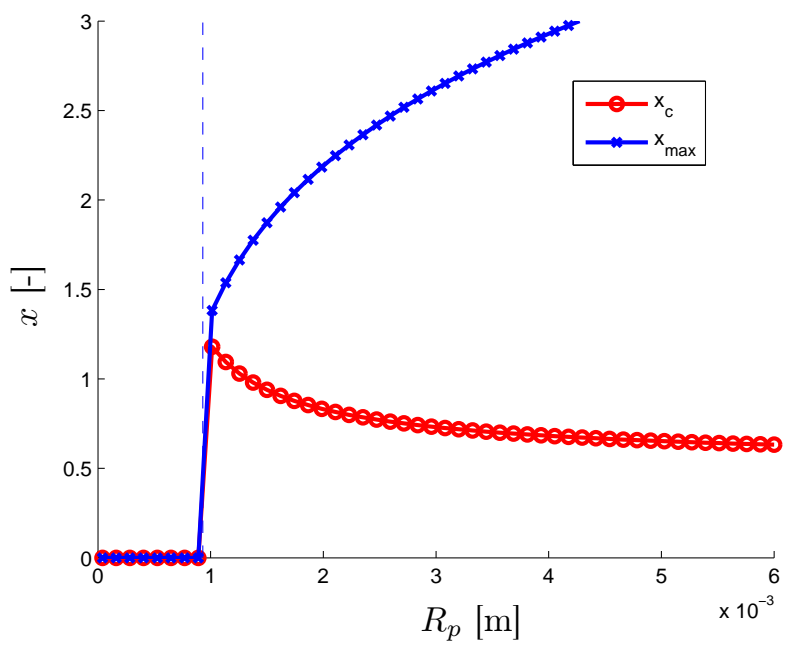

Figure 8: Relative initial $\left(x_{c}\right)$ and final $\left(x_{\max }\right)$ relative crack size as a function of aggregate size. $K_{\text {gel }}=1 \mathrm{GPa}$ and $\delta=1.03$

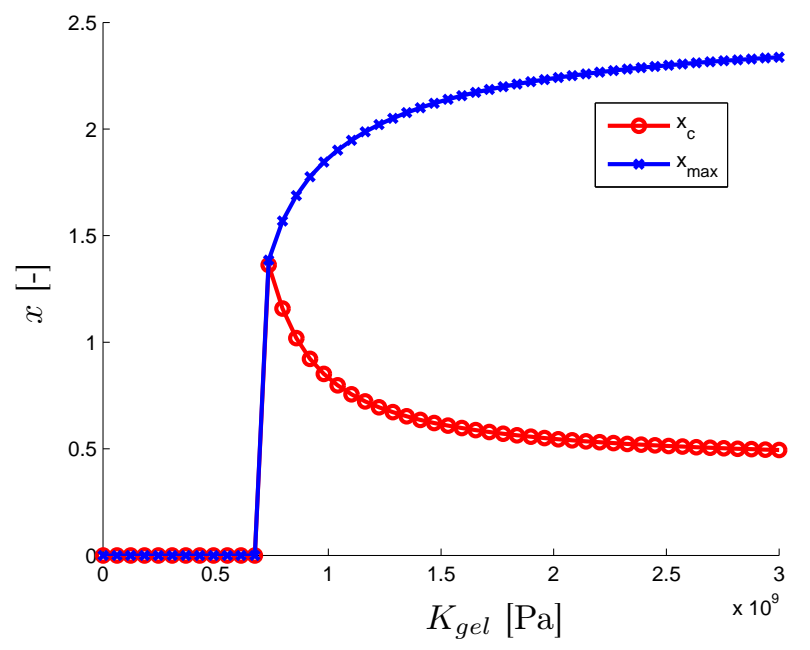

Figure 9: Relative initial $\left(x_{c}\right)$ and final $\left(x_{\max }\right)$ relative crack size as a function of the gel bulk modulus. $\delta=1.03$ and $R_{p}=1.5 \mathrm{~mm}$ 


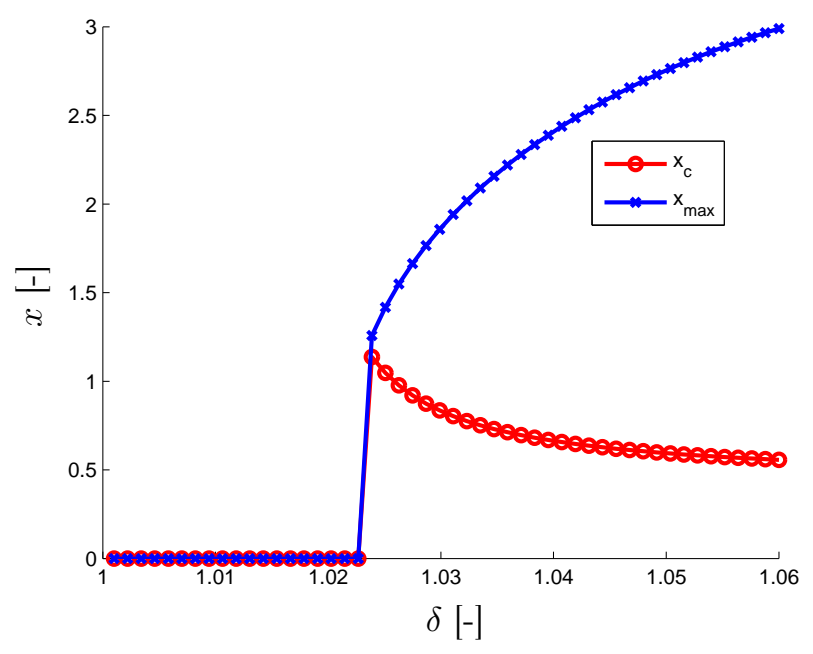

Figure 10: Relative initial $\left(x_{c}\right)$ and final $\left(x_{\max }\right)$ relative crack size as a function of the ratio of gel volume to aggregate volume. $K_{g e l}=$ $1 \mathrm{GPa}$ and $R_{p}=1.5 \mathrm{~mm}$

\subsubsection{Degree of attack corresponding to crack initiation when varying the aggregate radius}

Based on the energy criterion explained above (§ 5), we determine the degree of attack that leads to the initiation of a crack.

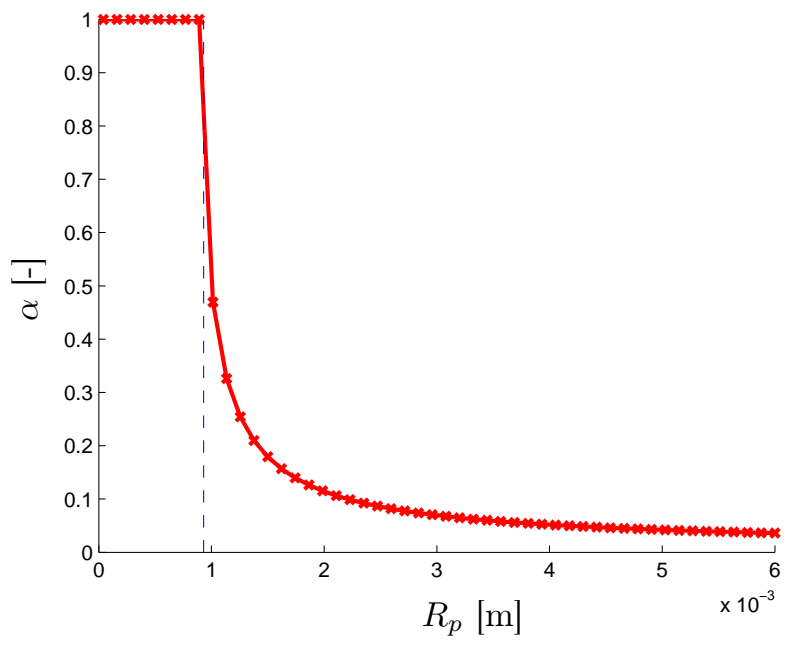

Figure 11: Degree of attack at first cracking. $K_{g e l}=1 \mathrm{GPa}$ and $\delta=1.03$

First, we see that the degree of attack at crack initiation decreases with increasing aggregate size (Fig. 11). The pressure at first cracking decreases with increasing aggregate size, as well as the pressure jump (Fig. 12). The order of magnitude of the pressure reached seems to be correct, thinking that Struble [27] has shown that synthetic gels can develop pressures of the order of $10 \mathrm{MPa}$, which is also the pressure chosen by Shin in his image-based FEA of alkali-silica reaction [25].

It is interesting to study the value of $\alpha R_{p}$ at first cracking for each aggregate size, since later in the article we as-

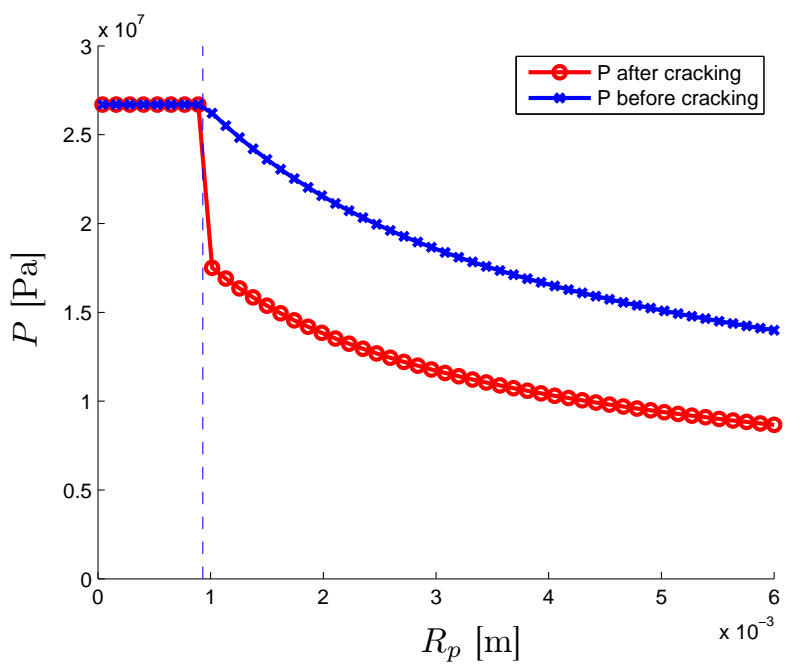

Figure 12: Pressure right before and after first cracking. $K_{g e l}=$ $1 \mathrm{GPa}$ and $\delta=1.03$

sume that when various sizes of aggregates are attacked simultaneously, the attack depth is the same for all (Fig. 13). In this case, a crack initiates around aggregates of intermediate size first, here around $3.6 \mathrm{~mm}$. We study as well the first cracking and final values of the absolute crack size $x R_{p}$. Very interestingly, once cracking has occurred, the absolute crack size is approximately affine with respect to the initial aggregate radius $R_{p}$ (Fig. 14).

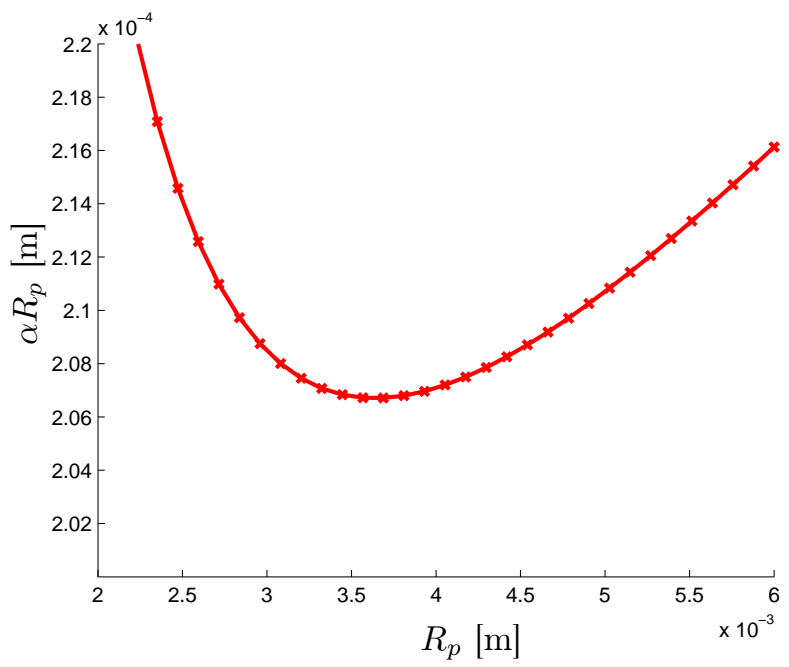

Figure 13: Attack depth at first cracking. $K_{\text {gel }}=1 \mathrm{GPa}$ and $\delta=1.03$ 


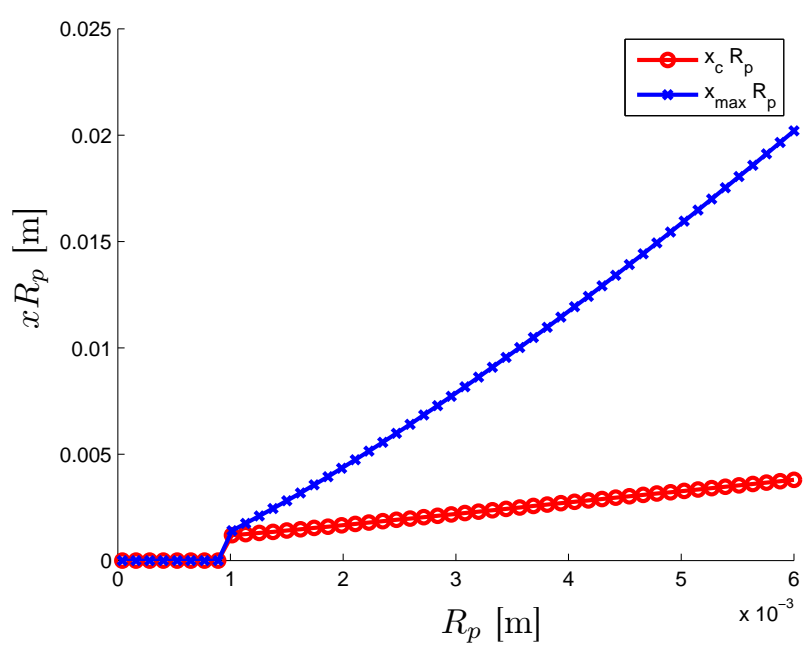

Figure 14: Absolute crack size at first cracking and at full attack of the aggregate. $K_{\text {gel }}=1 \mathrm{GPa}$ and $\delta=1.03$

7.2. Study of the complete evolution of the crack. Influence of an initial flaw

Once the crack is initiated, we can follow its evolution when degree of attack increases further. We still are in the approximation of a spherical attack, even if the presence of the crack increasingly leads this assumption to become wrong. In this article we mostly study the situation where there is no initial flaw (all crack sizes are initially zero), but we will show in this section a few evolutions of cracks with non-zero initial size.

For one aggregate size, here $R_{p}=1.5 \mathrm{~mm}$, we try different initial relative crack sizes, ranging from zero to the aggregate size. We represent the crack size $x$, the pressure $P$ and the volumetric deformation $\operatorname{tr} \underline{\underline{E}}$ as functions of $\alpha$, the relative attack depth. We consider 10 different cases: initial flaws ranging from $x=0$ (blue dotted line) to $x=1$.

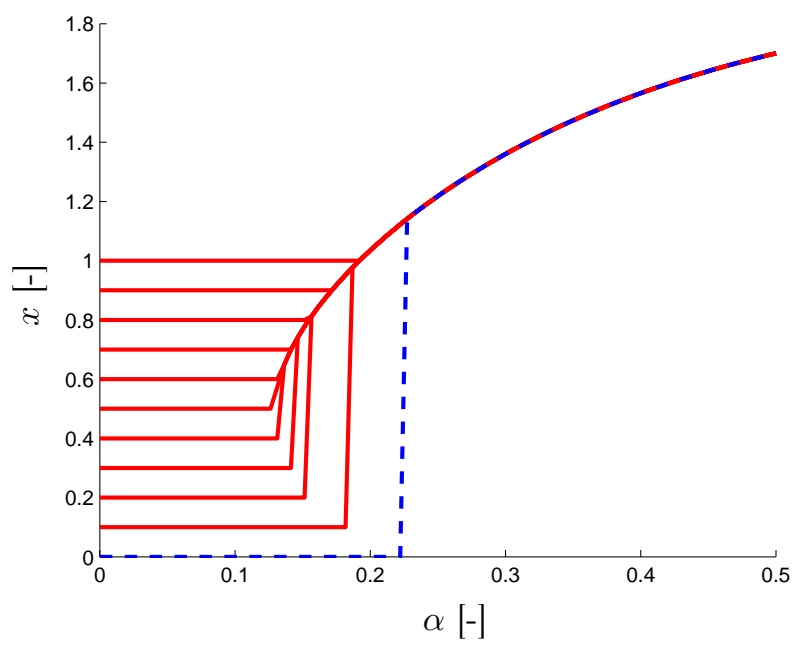

Figure 15: Relative crack size $x(\alpha)$ (initial flaws ranging from $x=0$ (dotted line) to $x=1$ ). $K_{\text {gel }}=1 \mathrm{GPa}, \delta=1.03$, and $R_{p}=1.5 \mathrm{~mm}$

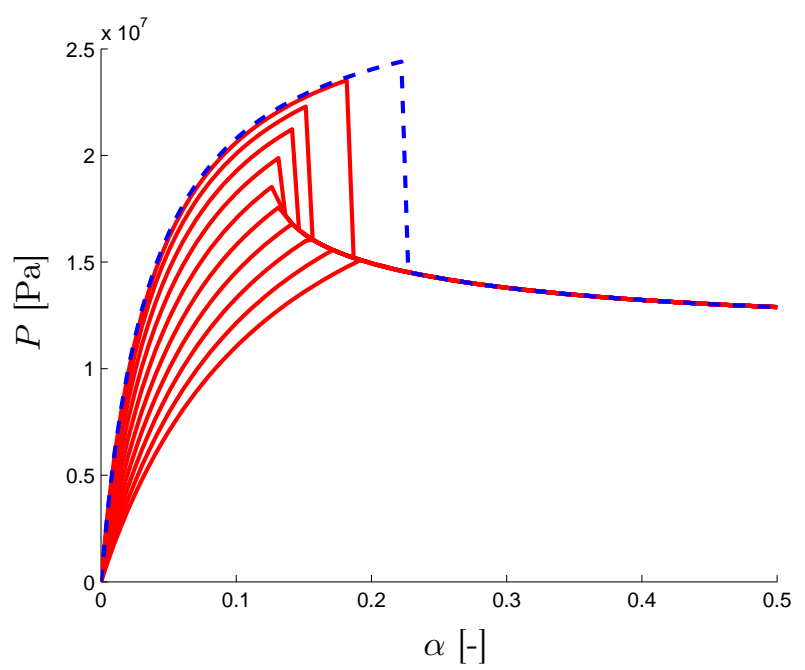

Figure 16: Pressure $P(\alpha)$ (initial flaws ranging from $x=0$ (dotted line) to $x=1) . K_{g e l}=1 \mathrm{GPa}, \delta=1.03$, and $R_{p}=1.5 \mathrm{~mm}$

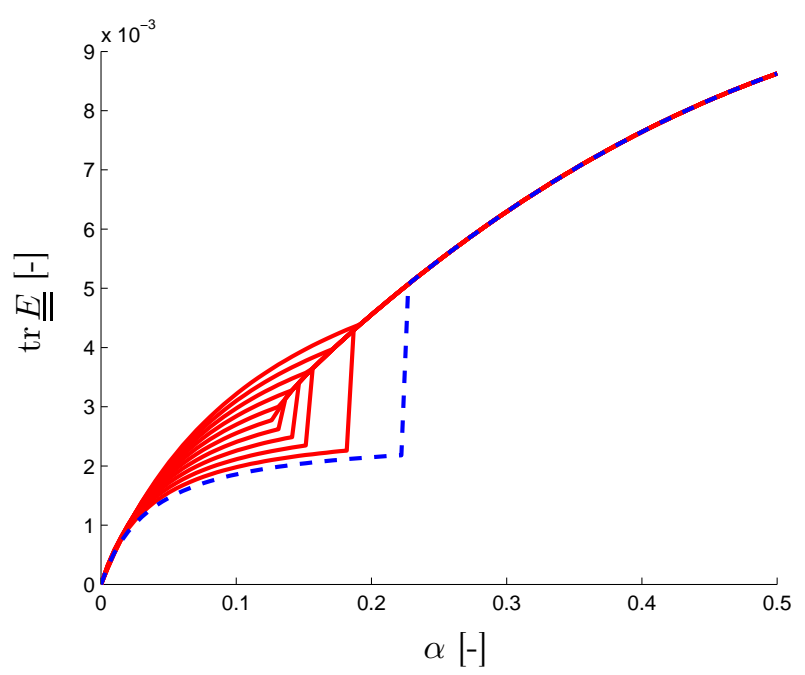

Figure 17: Volumetric deformation $\operatorname{tr} \underline{\underline{E}}(\alpha)$ (initial flaws ranging from $x=0$ (dotted line) to $x=1) . K_{\text {gel }}=1 \mathrm{GPa}, \delta=1.03$, and $R_{p}=1.5 \mathrm{~mm}$

We see the existence of a critical flaw size above which no jump in crack size occurs any more (Fig. 15), here approximatively $x=0.4$. Other simulations showed that this critical flaw size depends on the aggregate size. The smaller the flaw, the bigger the pressure has to be to propagate a crack (Fig. 16). If cracking occurs, expansion is ultimately the same, but a significantly different behaviour is observed at the beginning (Fig. 17). At early times, expansion is roughly doubled if a large flaw is considered, compared to the case with no initial crack. This effect seems very important here, when we only have one aggregate size involved. In next section, we discuss the same question with a full aggregate size distribution. 


\section{Numerical results: application to a given aggre- gate size distribution}

\subsection{Discussion about the existence of an original flaw}

Still focusing on cracking alone $\left(\rho=1, l_{c}=0\right)$, we plot the comparative evolution of the different aggregate sizes of a typical $0-4 \mathrm{~mm}$ Seine sand. Again we use the values $K_{\text {gel }}=1 \mathrm{GPa}$ (around half that of water) and $\delta=1.03$. We compare the case with no initial flaw to the case where around each aggregate exists an original flaw of size $x=0.5$ (chosen arbitrarily). See the cumulative volume fraction of this sand (Fig. 18). For these material properties, $R_{\text {inf }}=0.93 \mathrm{~mm}$, so some aggregates shall not induce cement paste cracking during this experiment.

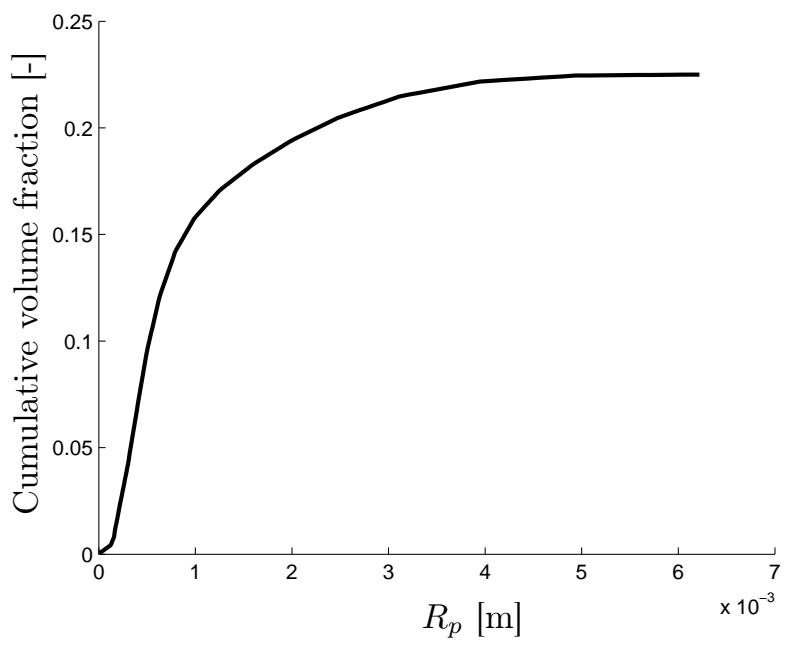

Figure 18: Seine sand

We plot the evolution of the crack size (Fig. 19) and the pressure (Fig. 20) for 5 aggregate sizes out of the 140 computed, and volumetric deformation (Fig. 21) for this Seine sand as functions of the attack degree $\alpha$. The curves corresponding to the case with an original flaw are the dashed curves.

When small aggregates are fully attacked and there was no original flaw, the pressure reaches the high value of $27 \mathrm{MPa}$ without fracture of the cement paste. The maximum pressure is slightly lower with initial flaws. Successive cracking leads to the irregularity of the expansion curve (Fig. 21) which is artificial since due to aggregate size discretization.

Finally, we see that the results are not too different. The expansion is slightly higher with initial flaws, because some aggregates were able to propagate an existing crack but were not able to create one from scratch. This is a good thing because it allows us to consider that there is no original flaw and hence to avoid the discussion about the size that should be given to this original flaw. This conclusion contrasts with the approach of Reinhardt in [22] where the original flaw size controls the fracture behaviour of aggregates submitted to ASR. Expansion is the fastest at the beginning of the attack. It progressively slows down.

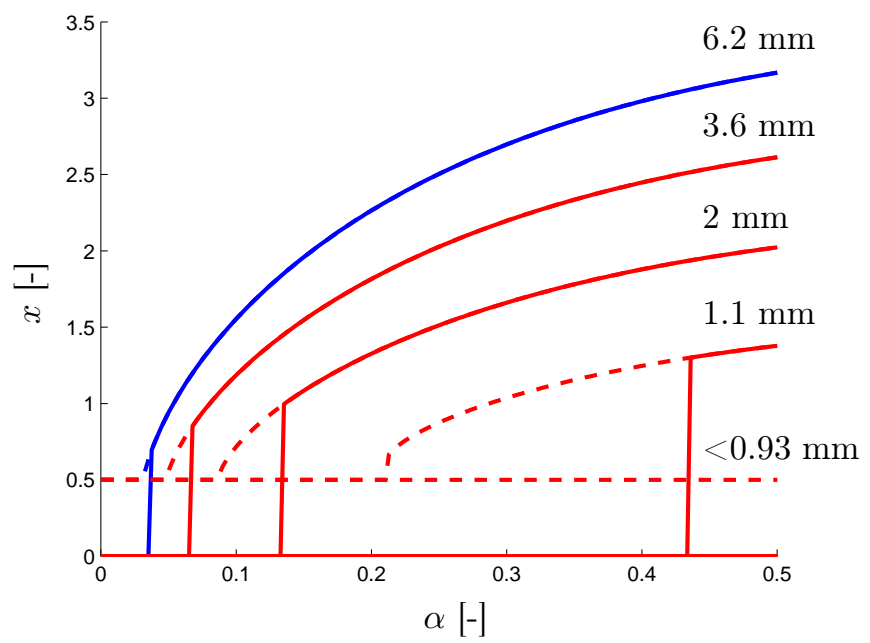

Figure 19: Evolution of crack size $x(t)$ for different aggregate sizes (written on the right of the plot) and initial crack lengths: $x=0$ (solid curves) and $x=0.5$ (dashed curves)

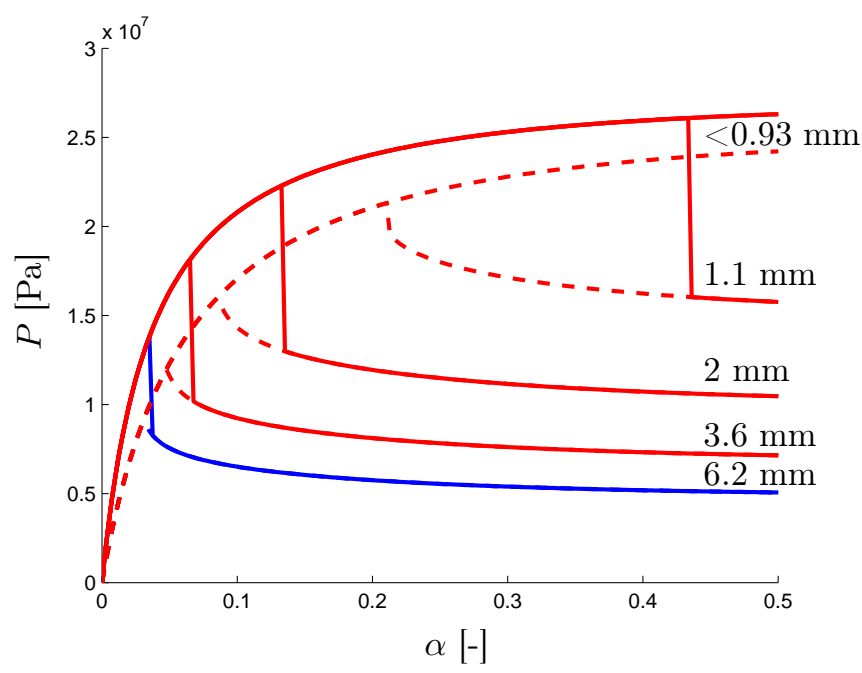

Figure 20: Pressure $p(t)$ for different aggregate sizes (written on the right of the plot) and initial crack lengths: $x=0$ (solid curves) and $x=0.5$ (dashed curves) 


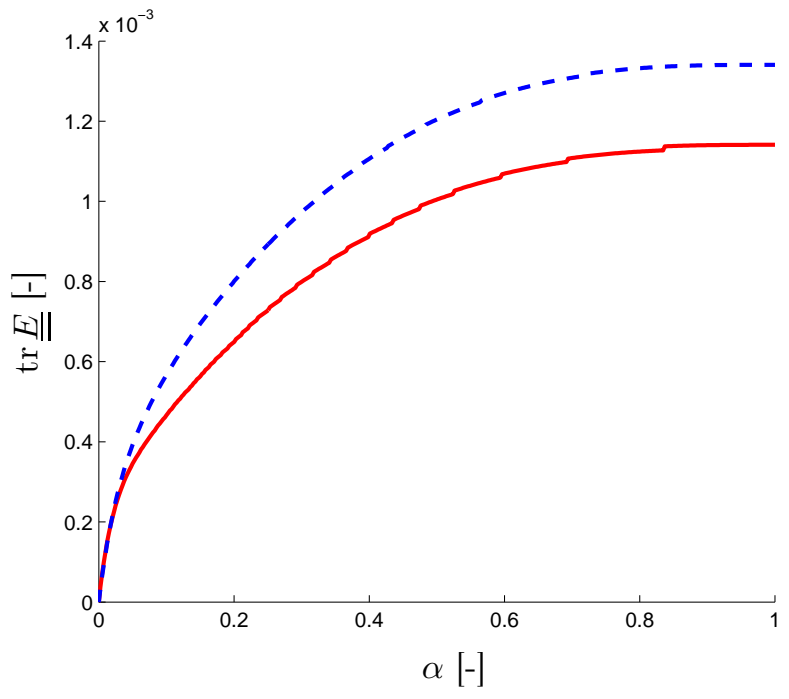

Figure 21: Cumulated volumetric expansion $\operatorname{tr} \underline{\underline{E}}$ for different initial crack lengths: $x=0$ (solid curve) and $x=0.5 \overline{\overline{(d}}$ ashed curve)

\subsection{Comparison with experimental ASR results}

\subsubsection{Experimental results used}

We must keep in mind that usual ASR expansion curves have a sigmoid shape characterized by a latency time, a characteristic time and an asymptotic expansion value [13]. We can wonder if our model can reproduce experimental results by identification of some of its parameters. We have chosen experimental data from Multon's article [18]. In this article the author presents a microscopic model and a set of swelling experiments of mortar bars containing different sizes of reactive sand. His model accounts for the diffusion of alkali in the aggregates whereas ours doesn't. He considers that no pressure is required to fill the ITZ with gel. The pressure build-up that occurs when the ITZ is full of gel leads to damage of the cement paste.

His mortars contain three aggregate size ranges:

- small: $80-160 \mu m, 30 \%$ mass of the aggregates

- medium: $315-630 \mu m, 40 \%$ mass of the aggregates

- large: 1250 - $3150 \mu \mathrm{m}, 30 \%$ mass of the aggregates

The reactive phase is siliceous limestone and is in the small or large size range in different proportions. In small aggregates, the reactive proportion of the aggregate is $9.4 \%$ mass. In the large ones, this proportion is $12.4 \%$. Hence for small aggregates we have $\rho=9.4$, for large aggregates $\rho=12.4$. We don't consider the experiments with mixed proportions of reactive aggregates, because our model is intrinsically unable to reproduce the couplings between different aggregate sizes. With our model, the expansion of a mix of two aggregate size is exactly the weighted average of the expansions of the two sizes alone, but his experiments show a more complex behaviour.

The total sand content is $1613.4 \mathrm{~kg} / \mathrm{m}^{3}$, the cement content $537.3 \mathrm{~kg} / \mathrm{m}^{3}$, the water to cement ration 0.5 , and the adjusted (by adding $\mathrm{NaOH}$ in the mix) alkali content is $6.2 \mathrm{~kg} / \mathrm{m}^{3}$ in the first set of experiments, $13.4 \mathrm{~kg} / \mathrm{m}^{3}$ in the second one.

In the 2 samples chosen for identification, first all the small fraction of aggregates is reactive, then all the large aggregates are reactive.

\subsubsection{Attack kinetics}

We have two choices to explain the end of expansion: scarcity of reactive phase in the aggregates, or scarcity of chemical products to attack the aggregates. The experimental results show a very important role of the quantity of alkali since the expansion is much faster and reaches greater asymptotic values with a greater initial alkali content. However since we don't model the chemical part of the reaction, we are not able to predict the slowing down of the reaction due to lack of alkali hydroxides.

Concerning the attack kinetics, following Bažant [2], we assume that at a given time, the attack depth is the same for all aggregate sizes.

$$
\alpha\left(R_{p}, t\right)=\frac{r(t)}{R_{p}}
$$

Then we need a simple assumption to determine the shape of $r(t)$. Assuming that the invasion of the aggregate by the interstitial solution is driven by diffusion, we choose the attack depth common to all aggregates to be proportional to the square root of time (also following Multon [16] on this point):

$$
r(t)=f_{r} \sqrt{t}
$$

\subsubsection{Optimization}

We then perform a least square optimization procedure over 5 parameters:

- the bulk modulus $K_{g e l}$ of the gel, which is unknown and might depend on gel composition

- the expansion coefficient $\delta$ of the gel, which is also unknown and might depend on gel composition

- the thickness of the expansion reservoir taking the ITZ into account $l_{c}$ which shall not depend on the type of gel

- the pressure $P_{0}$ at which the ITZ is considered to be full, which is a mixed property of the ITZ and the gel

- the coefficient $f_{r}$, which represents the speed of the attack. It might change when the alkali concentration changes, but not with aggregate size

Therefore, we first identify 5 parameters using the two expansion curves at $6.2 \mathrm{~kg} / \mathrm{m}^{3}: f_{r}^{6.2}, K_{\text {gel }}^{6.2}, \delta^{6.2}, P_{0}$, and $l_{c}$. Then we fix the two last parameters $P_{0}$ and $l_{c}$, and identify the 3 remaining unknowns: $f_{r}^{13.4}, K_{\text {gel }}^{13 .}, \delta^{13.4}$.

The fits (see Fig. 22 and Fig. 23) are obtained with the following values of the parameters: $f_{r}^{6.2}=0.66 \max (R p)$, 


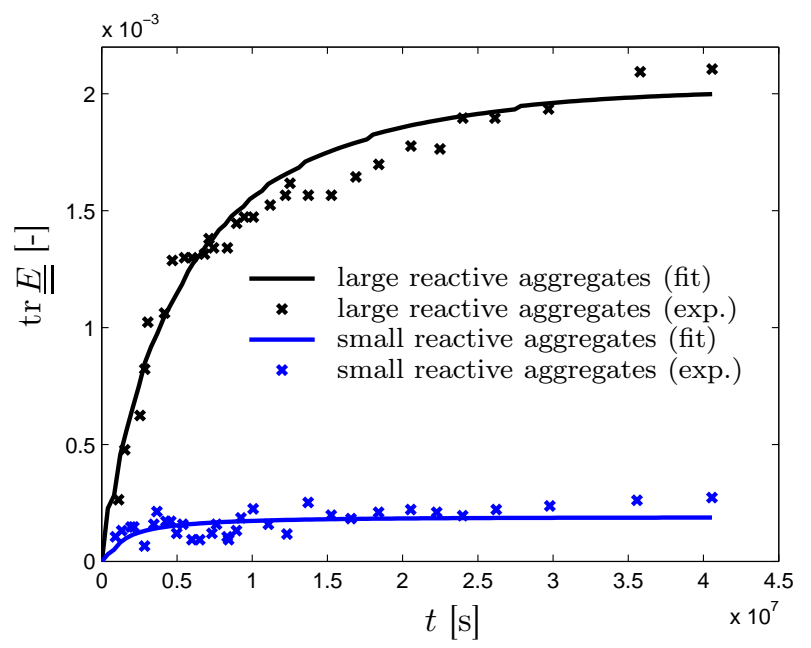

Figure 22: Fit of the expansion curves obtained with a total alkali content of $6.2 \mathrm{~kg} / \mathrm{m}^{3}$

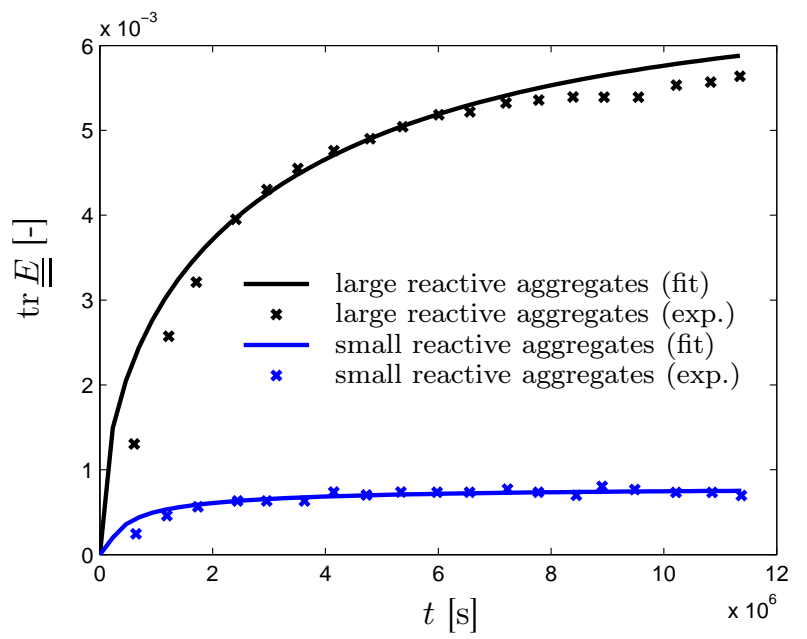

Figure 23: Fit of the expansion curves obtained with a total alkali content of $13.4 \mathrm{~kg} / \mathrm{m}^{3}$

$K_{\text {gel }}^{6.2}=70 \mathrm{MPa}, \delta^{6.2}=1.4, P_{0}=2.7 \mathrm{MPa}, l_{c}=0.42 \mu \mathrm{m}$, $f_{r}^{13.4}=0.4 \max (R p), K_{\text {gel }}^{13.4}=183 \mathrm{MPa}$, and $\delta^{13.4}=1.7$. The expansion curves related to the greater alkali content (Fig. 23) is less good, probably because it was performed second, using values of $P_{0}$ and $l_{c}$ identified on the two other curves.

More physics is needed. The greater quantity of alkali leads to a higher expansion. Since we don't model the alkali, the increase in asymptotic expansion is obtained by an increase of $K_{\text {gel }}$ and $\delta$. Moreover, since no experiments are available concerning the swelling and elastic properties of gels formed at different alkali content, we don't know if the large variations of $K_{g e l}$ and $\delta$ keep with the physics of real gels. Some experiments are in progress concerning their stiffness. We hope to be able to comment the values identified here in a further article. To limit initial expansion, $f_{r}$ (which is expressed relatively to the greatest aggregate size) is lead to decrease, but the initial expansion is still overestimated.

With both alkali contents, no crack appears around the smallest fraction of aggregates. For the large aggregates, it reaches sizes as large as $x=4$, which is a few millimetres depending on the aggregate size.

\subsubsection{Pessimum size behaviour}

Pessimum effects are systematically observed with ASR. Both the aggregate total volume fraction and size distribution play an important role. Many existing models can predict part of these pessimum effects. Concerning our model:

- The pessimum concentration effect, according to which there is an aggregate volume fraction that leads to maximum expansion cannot be reproduced since the volume fraction of aggregates only plays a role in a summation, there is no notion of chemistry presently in our model, so no risk of scarcity of reactants to attack the aggregate or produce the gel. Even assuming for example that only a certain volume of aggregate can react and testing different total volume fraction, we obtain no total concentration pessimum effect.

- A transitory pessimum size effect is observed with our model. We study this effect on a granulometry ranging from 0 to $5 \mathrm{~mm}$ (no important pessimum size effect can be expected if all aggregate sizes are over $R_{\text {inf }}$, since the effect of earlier cracking of middlesized aggregates is very short-lasting). We still are in the assumption that the attack depth is the same over all aggregate sizes at a given time. Here, we have a transitory pessimum effect related to the fact that at a given experiment time (that is in our model at a given attack depth), different aggregate sizes lead to different expansions (at identical volume fraction) (Fig. 24). Starting from the bottom, each curve correspond to a given attack time. The black solid curve represents full attack while other curves (from bottom to top) are spaced by increments of $5 \%$ relatively to this final time. As we can see, the pessimum size is roughly around $2 \mathrm{~mm}$ and varies during the experiment. Reaching greater attack times, no more pessimum size exists: bigger aggregates are responsible for bigger expansion, at equal volume fraction. Notice the resemblance of our description of the pessimum size effect to Multon's [16]. The main difference is that in our model, the smallest aggregates lead to non-zero expansion since some pressure is required to fill the ITZ. Therefore even if the cement paste is uncracked, we predict some expansion. 


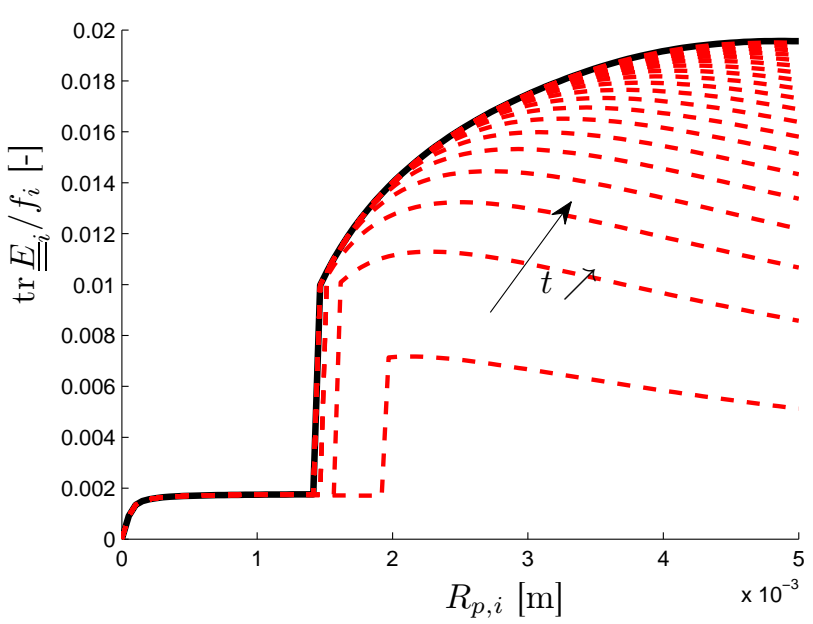

Figure 24: Pessimum size effect: expansion due to a given aggregate size at unit volume fraction. $K_{\text {gel }}=70 \mathrm{MPa}, \delta=1.4, P_{0}=2.7 \mathrm{MPa}$, $l_{c}=0.42 \mu \mathrm{m}$, and $R_{p} \in[0 \mathrm{~mm} ; 5 \mathrm{~mm}]$. Each curve represent a given instant by $5 \%$ time increments relatively to final time (solid curve)

\section{Conclusion}

A model for Alkali-reaction was proposed. It is a strictly mechanical model, partly inspired by that of Bažant [2] and [18]. Aggregates are supposed to behave as if they were embedded in an infinite cement paste. The chemical attack is modelled by progressively replacing the aggregate by a less dense gel. The subsequent pressure increase leads to cracking of the surrounding cement paste. The initiation and propagation of the crack are governed by energy balance only.

An interesting behaviour is observed. Aggregates of different sizes have a different impact on the overall expansion. First of all, even fully attacked, smaller aggregates are shown not to lead to any cracking of the cement paste. The pressure reached for these aggregates is important, but according to our energy criterion the cement paste can take such pressures when there is no (or a very small) initial crack, because the elastic energy stored in the cement paste around a small aggregate is not sufficient to provide the energy needed to create a crack.

The model is then used to discuss the importance of the initial flaw considered. We show that even if the presence and the size of an initial flaw is important for the expansion due to a single aggregate size, after summation of different sizes, the effect is not enormous. This conclusion is linked to the fact that we only considered an energetic fracture criterion.

Finally we apply the model to try to reproduce the expansion obtained by Multon [18] and identify the parameters introduced. No attempt was made to predict other expansion curves using the identified parameters because we are aware that some physics still lacks for the model to be predictive.

\section{References}

[1] Bažant, Z. P., Steffens, A., 2000. Mathematical model for kinetics of alkali-silica reaction in concrete. Cement and Concrete Research 30, $419-428$.

[2] Bažant, Z. P., Zi, G., Meyer, C., 2000. Fracture mechanics of ASR in concretes with waste glass particles of different sizes. Journal of Engineering Mechanics 126-3, 226-232.

[3] Ben Haha, M., 2006. Mechanical effects of alkali silica reaction in concrete studied by SEM-image analysis. Ph.D. thesis, EPFL, Suisse.

[4] Ben Haha, M., Gallucci, E., Guidoum, A., Scrivener, K., 2007. Relation of expansion due to alkali silica reaction to the degree of reaction measured by SEM image analysis. Cement and Concrete Research 37, 1206 - 1214.

[5] Capra, B., Bournazel, J.-P., 1998. Modeling of induced mechanical effects of alkali-aggregate reaction. Cement and Concrete Research 28, 251-260.

[6] Dormieux, L., Lemarchand, E., Kondo, D., Fairbairn, E., 2004. Elements of poro-micromechanics applied to concrete. Materials and Structures 37, $31-42$.

[7] Dunant, C., 2009. Experimental and modelling study of the alkali-silica reaction in concretes. Ph.D. thesis, EPFL, Suisse.

[8] Dunant, C. F., Scrivener, K. L., 2010. Micro-mechanical modelling of alkali-silica-reaction-induced degradation using the AMIE framework. Cement and Concrete research 40, $517-$ 525.

[9] Gaboriaud, F., Nonat, A., Chaumont, D., Craievich, A., 2005. Structural model of gelation processes of a sodium silicate sol destabilized by calcium ions: combination of SAXS and rheological measurements. Journal of Non-Crystalline Solids 351, 351-354.

[10] Giaccio, G., Zerbino, R., Ponce, J., Batic, O., 2008. Mechanical behavior of concretes damaged by alkali-silica reaction. Cement and Concrete Research 38, 993 - 1004.

[11] Ichikawa, T., 2009. Alkali-silica reaction, pessimum effects and pozzolanic effects. Cement and Concrete Research 39, 716 - 726.

[12] Ichikawa, T., Miura, M., 2007. Modified model of alkali-silica reaction. Cement and Concrete Research 37, 1291 - 1297.

[13] Larive, C., 1997. Apports combinés de l'expérimentation et de la modélisation à la compréhension de l'alcali-réaction et de ses effets mécaniques. Ph.D. thesis, École Nationale des Ponts et Chaussées, France.

[14] Lemarchand, E., Dormieux, L., Fairbairn, E., Ribiero, F., 2005. A micromechanical approach to ASR-induced damage in concrete. Third Biot Conference, Norman, Oklahoma, USA.

[15] Lemarchand, E., Dormieux, L., Ulm, F.-J., 2005. Micromechanics investigation of expansive reactions in chemoelastic concrete. Philosophical Transactions of the Royal Society A 363, 2581 2602.

[16] Multon, S., Cyr, M., Sellier, A., Diederich, P., Petit, L., 2010. Effects of aggregate size and alkali content on ASR expansion. Cement and Concrete Research 40, 508 - 516.

[17] Multon, S., Cyr, M., Sellier, A., Leklou, N., Petit, L., 2008. Coupled effects of aggregate size and alkali content on ASR expansion. Cement and Concrete Research 38, 350 - 359.

[18] Multon, S., Sellier, A., Cyr, M., 2009. Chemo-mechanical modeling for prediction of alkali silica reaction(ASR) expansion. Cement and Concrete Research 39, 490 - 500.

[19] Phair, J. W., Tkachev, S. N., Manghani, M. H., Livingston, R. A., 2005. Elastic and structural properties of alkaline-calcium silica hydrogels. Journal of Materials Research 20 - 2, $344-349$.

[20] Ponce, J., Batic, O., 2006. Different manifestations of the alkalisilica reaction in concrete according to the reaction kinetics of the reactive aggregates. Cement and Concrete Research 36, 1148 $-1156$.

[21] Poyet, S., 2003. étude de la dégradation des ouvrages en béton atteints par la réaction alcali-silice: Approche expérimentale et modélisation numérique multi-échelles des dégradations dans un environnement hydro-chemo-mécanique variable. Ph.D. thesis, Université de Marne-La Vallée, France. 
[22] Reinhardt, H., Mielich, O., 2011. A fracture mechanics approach to the crack formation in alkali-sensitive grains. Cement and Concrete Research 41, 255 - 262.

[23] Riche, J., 2003. La réaction alcali-silice : approche cinétique et mécanisme d'expansion. étude du système silex-chaud-potasse à $80^{\circ} \mathrm{c}$. Ph.D. thesis, Université de Lille, France.

[24] Sellier, A., 1995. Modélisations probabilistes du comportement de matériaux et de structures en génie civil. Ph.D. thesis, Ecole Normale Supérieure de Cachan, France.

[25] Shin, J.-H., 2009. Modelling alkali-silica reaction using image analysis and finite element method. Ph.D. thesis, University of Illinois at Urbana-Champaign, USA.

[26] Stanton, T., 1940. Expansion of concrete through reaction between cement and aggregate. Proceedings of the American Society of Civil Engineering 66-10, $1781-1811$.

[27] Struble, L. J., Diamond, S., 1981. Swelling properties of synthetic alkali silica gels. Journal of the American Ceramic Society 64-11, $652-655$.

[28] Suwito, A., Jin, W., Xi, Y., Meyer, C., 2002. A mathematical model for the pessimum size effect of asr in concrete. Concrete Science and Engineering 4-13, 23 - 34.

[29] Tanaka, K., Mori, T., 1972. Note on volume integrals of the elastic field around an ellipsoidal inclusion. Journal of Elasticity $2,199-200$.

[30] Wittmann, F., 2002. Crack formation and fracture energy of normal and high strength concrete. Sādhanā 27 - 4, 413 - 423.

[31] Xiao, Z., J.Y., G., S., Y., 1999. Stress intensity factors for ringshaped crack surrounded by spherical inclusions. Theoretical and applied fracture mechanics 32, $147-155$. 